\title{
Fault Diagnosis of Rolling Bearing Based on a Novel Adaptive High-Order Local Projection Denoising Method
}

\author{
Rui Yuan $\mathbb{D}^{1,2}$ Yong $\mathrm{Lv} \mathbb{D}^{1,2}$ and Gangbing Song $\mathbb{D}^{3}$ \\ ${ }^{1}$ Key Laboratory of Metallurgical Equipment and Control Technology, Wuhan University of Science and Technology, \\ Ministry of Education, Wuhan 430081, China \\ ${ }^{2}$ Hubei Key Laboratory of Mechanical Transmission and Manufacturing Engineering, Wuhan University of Science and Technology, \\ Wuhan 430081, China \\ ${ }^{3}$ Smart Material and Structure Laboratory, Department of Mechanical Engineering, University of Houston, Houston, TX 77204, USA \\ Correspondence should be addressed to Yong Lv; lvyong@wust.edu.cn and Gangbing Song; gsong@uh.edu
}

Received 20 January 2018; Revised 3 August 2018; Accepted 6 September 2018; Published 11 October 2018

Academic Editor: Marcin Mrugalski

Copyright (C) 2018 Rui Yuan et al. This is an open access article distributed under the Creative Commons Attribution License, which permits unrestricted use, distribution, and reproduction in any medium, provided the original work is properly cited.

\begin{abstract}
Rolling bearings are vital components in rotary machinery, and their operating condition affects the entire mechanical systems. As one of the most important denoising methods for nonlinear systems, local projection (LP) denoising method can be used to reduce noise effectively. Afterwards, high-order polynomials are utilized to estimate the centroid of the neighborhood to better preserve complete geometry of attractors; thus, high-order local projection (HLP) can improve noise reduction performance. This paper proposed an adaptive high-order local projection (AHLP) denoising method in the field of fault diagnosis of rolling bearings to deal with different kinds of vibration signals of faulty rolling bearings. Optimal orders can be selected corresponding to vibration signals of outer ring fault (ORF) and inner ring fault (IRF) rolling bearings, because they have different nonlinear geometric structures. The vibration signal model of faulty rolling bearing is adopted in numerical simulations, and the characteristic frequencies of simulated signals can be well extracted by the proposed method. Furthermore, two kinds of experimental data have been processed in application researches, and fault frequencies of ORF and IRF rolling bearings can be both clearly extracted by the proposed method. The theoretical derivation, numerical simulations, and application research can indicate that the proposed novel approach is promising in the field of fault diagnosis of rolling bearing.
\end{abstract}

\section{Introduction}

Rolling bearings are of vital importance in rotary machinery systems, and they are prone to failures due to the complex running conditions [1]. The performance of rolling bearings can affect the reliability of operation of the entire system directly. Therefore, it is of great significance to conduct fault diagnosis of rolling bearing. At present, structural health monitoring of rolling bearing usually relies on analyzing its vibration signal [2-4]. During the operation of mechanical systems, the machine is usually accompanied by strong nonlinear and nonstationary vibrations, and the rolling bearing failure will further cause the unexpected vibration of other components, so the measured vibration signals are generally mixed with or submerged in vibration signal of other parts and background noise $[5,6]$. The denoising of vibration signal of faulty rolling bearing contributes to extracting the fault frequency of the faulty rolling bearing, to achieve the fault identification and diagnosis of rolling bearing, while the vibration signals collected from mechanical systems usually have nonlinear and nonstationary characteristics, which make denoising methods based on linear systems unsuitable $[7,8]$. The rolling bearing vibration signals collected from operating mechanical systems are usually taken as chaotic signals $[9,10]$. For nonlinear time series, useful information would be mistakenly filtered out if traditional linear denoising methods are conducted, which will subsequently result in distortion and deformation of the original signal [11]. There are some methods developed for nonlinear and nonstationary signal analysis, such as wavelet packet decomposition (WPD) [12-14], singular value decomposition (SVD) $[15,16]$, and ensemble empirical mode decomposition 
(EEMD) [17]. While they rely on characteristic frequency components of the collected signal to some extent, however, it is difficult to extract the fault frequencies if the rolling bearing has incipient fault. On that occasion, the fault frequencies of rolling bearing would be submerged in all disturbing frequencies.

The denoising method based on phase space reconstruction has gradually become one of the most important tools to research nonlinear and nonstationary signals. A time series can be reconstructed into high-dimensional phase space through delay embedding, and the reconstructed phase space is diffeomorphic to original dynamic system, i.e., they have the same dynamic characteristics [18-21]. The dynamic characteristics contained in one-dimensional nonlinear time series can be revealed and extracted by studying the motion characteristics and distribution of attractor in high-dimensional phase space. The advantage of highdimensional signal processing has also been verified in the fields biomedical science and communication engineering $[22,23]$. The noise can be filtered out by adopting different projection ways of high-dimensional phase space. For instance, smooth orthogonal decomposition (SOD) [24-26] projects the points in a high-dimensional phase space into different subspaces to reduce noise, and characteristic frequencies of the original time series can be extracted. The main idea of the local projection algorithm was originally proposed to conduct noise reduction $[27,28]$. It projects the points in the neighborhood onto the attractor, correcting the raw data by projecting into a few principle directions, which are preferred by locally smoothed data. The local projective filter method was improved to correct the location of points in phase space by determining locations of neighboring points [29]. The noise is reduced by requiring locally linear relations among the delay coordinates and moving delay vectors closer to smooth manifolds. Then, the local projective filters had been employed in spheres ranging from biology signal analysis [30] to space signal analysis [31]; the general framework of local projection was adopted to analyze signals from particular systems, such as being applied to remove interferential artifacts from an electroencephalography (EEG) signal among them. A local projection stabilized and characteristic decoupled scheme aiming at the fluid-fluid interaction problems was proposed. It can reflect the extensive applications of the local projective method [32]. More recently, the parameters of the local projection filtering method were optimized and applied to noisily observed variations in light intensity between the star and its observer [33]. Subspace was proposed to eliminate noise subsequently, and the sequences of temporally successive points in phase space are projected to the relative position of neighboring strands [24-26]. Among SOD, noises in the data are reduced by projecting to the tangent subspace. The vector directions of the projection can make sure the variance as maximum as possible, and the motions obtaining along these vector directions are the smoothest in terms of time.

The centroid selection of local neighborhoods is of vital importance in the local projection method to estimate the neighborhood correctly. The first-order statistics and the
TABLE 1: The algorithm of standard LP denoising method.

(1) Select appropriate embedding dimension $m$ and delay time $\tau$ to reconstruct noisy time series into $m$ dimensional phase space.

(2) Determine neighborhood $U_{n}$ for one phase point.

(3) Calculate centroid of its neighborhood by fixed neighborhood numbers.

(4) Calculate covariance matrix $\mathbf{C}$ of its phase point neighborhood and then conduct eigenvalue decomposition to obtain eigenvector $\mathbf{a}_{q}$ corresponding to $m-m_{0}$ smaller eigenvalues. $\left(q=1,2, \ldots, m-m_{0}\right)$.

(5) Subtract the projection of the phase point in noise subspace by $\mathbf{X}_{n}{ }^{\prime}=\mathbf{X}_{n}-\mathbf{R}^{-1} \sum_{q=1}^{m-m_{0}} \mathbf{a}_{q}\left[\mathbf{a}_{q} \cdot \mathbf{R}\left(\mathbf{X}_{n}-\overline{\mathbf{X}}_{n}\right)\right]$

where $\mathbf{R}$ is a diagonal weight matrix, aiming at inhibiting the distortion of ending elements of phase points and reserving the middle stable elements $\left(\mathbf{R}_{11}\right.$ and $\mathbf{R}_{m m}$ were set as $10^{3} ; \mathbf{R}_{i i}$ values were set as 1 in our research). $\overline{\mathbf{X}}_{n}$ is the centroid of neighborhood; $X_{n}^{\prime}$ denotes the denoised signal.

(6) Get back to step (2) and repeat subsequent steps until all phase points are processed.

second-order statistics have been widely used in the analysis of mechanical vibration signals, and these methods are theoretically applicable to the analysis of linear and Gaussian signal [29, 34, 35]. While for nonlinear and nonstationary signals, higher orders can be utilized to better reflect the signal characteristics, and high-order polynomials have been used to estimate the centroid of neighborhood among LP denoising method in the field of medical signal processing [36] and thermodynamics [37]. Compared with LP denoising method employing the first- and the second-order statistics, HLP denoising method employing higher-order statistics has the advantages in reducing noise and extracting characteristic frequencies, by adapting to phase space nonlinearity. The extension to HLP denoising method can reduce noise more effectively than previous LP filtering [34-37]. In this paper, by employing high-order polynomials to calculate the centroid of neighborhood during the LP procedures, the HLP was firstly adopted to denoise the vibration signals of faulty rolling bearing to extract its fault characteristic frequencies.

This paper proposes a novel approach called AHLP denoising method aiming at fault diagnosis of rolling bearings. In the proposed AHLP denoising method, except adopting high-order polynomials to calculate the centroid of neighborhood, optimal orders of dealing with different kinds of rolling bearing faults can be further estimated to achieve a better denoising effect. The AHLP denoising method can better denoise the vibration signal of faulty rolling bearing to extract its fault characteristic frequencies among all disturbing frequencies, which contributes to the field of fault diagnosis of rolling bearing. The organization of this paper is as follows: Section 2 introduces the methodologies of standard LP, HLP, and AHLP denoising methods and illustrates the scheme of AHLP denoising method in 


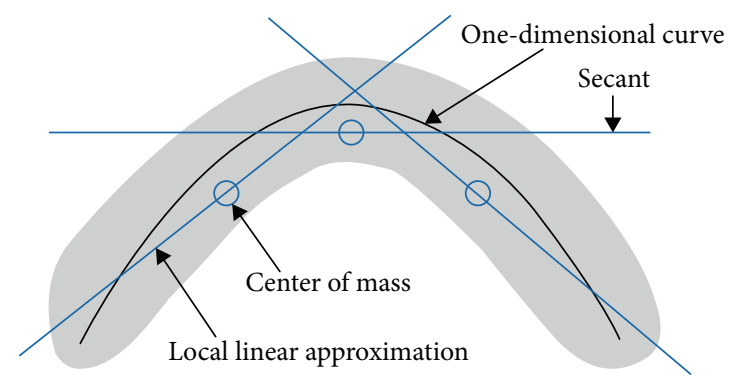

(a)

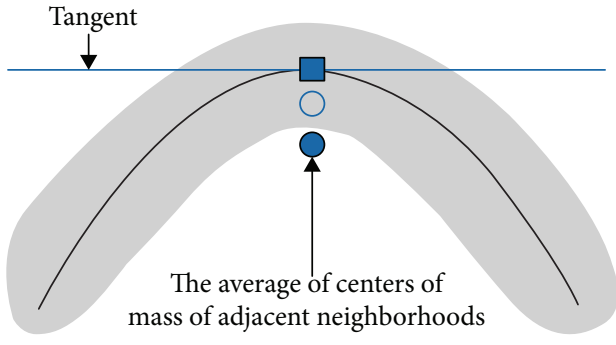

(b)

FIGURE 1: Consider a cloud of points around a curve. (a) The mean of phase points of neighborhood is estimated as centroid of neighborhood. (b) The second-order polynomial is used to estimate the centroid of neighborhood.

TABLE 2: The $a_{i}$ value of different orders in HLP denoising method.

\begin{tabular}{|c|c|c|c|c|c|c|c|c|c|c|}
\hline 1 & & & & & & & & & Order & $a_{i}$ \\
\hline 1 & 1 & & & & & & & & 1 & {$[1]$} \\
\hline 1 & 2 & 1 & & & & & & & 2 & {$[2,-1]$} \\
\hline 1 & 3 & 3 & 1 & & & & & & 3 & {$[3,-3,1]$} \\
\hline 1 & 4 & 6 & 4 & 1 & & & & & 4 & {$[4,-6,4,-1]$} \\
\hline 1 & 5 & 10 & 10 & 5 & 1 & & & & 5 & {$[5,-10,10,-5,1]$} \\
\hline 1 & 6 & 15 & 20 & 15 & 6 & 1 & & & 6 & {$[6,-15,20,-15,6,-1$} \\
\hline 1 & 7 & 21 & 35 & 35 & 21 & 7 & 1 & & 7 & {$[7,-21,35,-35,21,-7,1]$} \\
\hline 1 & 8 & 28 & 56 & 70 & 56 & 27 & 8 & 1 & 8 & {$[8,-28,56,-70,56,-28,-1]$} \\
\hline
\end{tabular}

fault diagnosis of rolling bearing. Section 3 introduces the vibration signal model of faulty rolling bearings and presents numerical simulations including simulated vibration signals of ORF and IRF rolling bearings. By choosing different orders to denoise simulated signals and extract characteristic frequencies, the optimal orders are obtained. Section 4 presents the applications to bearing monitoring with two different practical experimental signals to validate the proposed method. The conclusions of the researches and necessary discussions are given in Section 5.

\section{Methodology}

2.1. Standard Local Projection Denoising Method. Assuming that $x(1), x(2), \ldots, x(N) \in R$ is a time series of length $N$ extracted from a chaotic system, then it can be embedded in phase space with appropriate embedding dimension and delay time based on the embedding theorem [18-20]. The reconstructed phase space has the same diffeomorphism as the original time series, namely, they have the same dynamic characteristics. The reconstructed phase space can be expressed as follows:

$$
\mathbf{X}_{n}=\left(x_{n-(m-1) \tau}, x_{n-(m-2) \tau}, \ldots, x_{n}\right)
$$

where $\mathbf{X}_{n}$ denotes the $n$th phase point in reconstructed phase space, $m$ is the embedding dimension, and $\tau$ is the delay time. The minimum embedding dimension $m$ needs to meet the criterion $m \geq 2 d+1$, when fractional dimension of system attractor is $d$. Under this circumstance, the reconstructed phase space is diffeomorphic to the original dynamic system, namely, they have the same dynamic characteristics. In $m$ dimensional phase space, the attractor that reflects dynamic behavior of system is usually confined to a low-dimensional subspace of $m_{0}\left(d<m_{0}<m\right)$. When there is no noise interference, zero subspace of $m-m_{0}$ exists. When noise is present, it is randomly distributed in phase space, and the components from subspace of $m-m_{0}$ are generated by noise. For each phase point, eigenvalue decomposition is conducted to deal with covariance matrix composed by all phase points within neighborhood. The subspaces corresponding to $m_{0}$ larger eigenvalues are taken as signal subspace, while other $m-m_{0}$ smaller eigenvalues correspond to the noise subspace. The LP denoising method is aimed at finding the abovementioned noise subspace and then subtract the projection of phase points in this subspace. The algorithm of the LP method is given in Table 1.

During step (1), embedding dimension $m$ and delay time $\tau$ are critical parameters. The bigger embedding dimension $m$ contributes to overembedding to fully extract the characteristics of the time series, but meanwhile, it decreases the computational efficiency. The smaller time delay $\tau$ can make neighborhood points closer to the attractor. Hence, in this paper, the mutual information method [38] is adopted to determine delay time $\tau$, and then the proposed method by CAO [39] is used to determine embedding dimension $m$. As for step (3) of standard LP denoising method, the method 


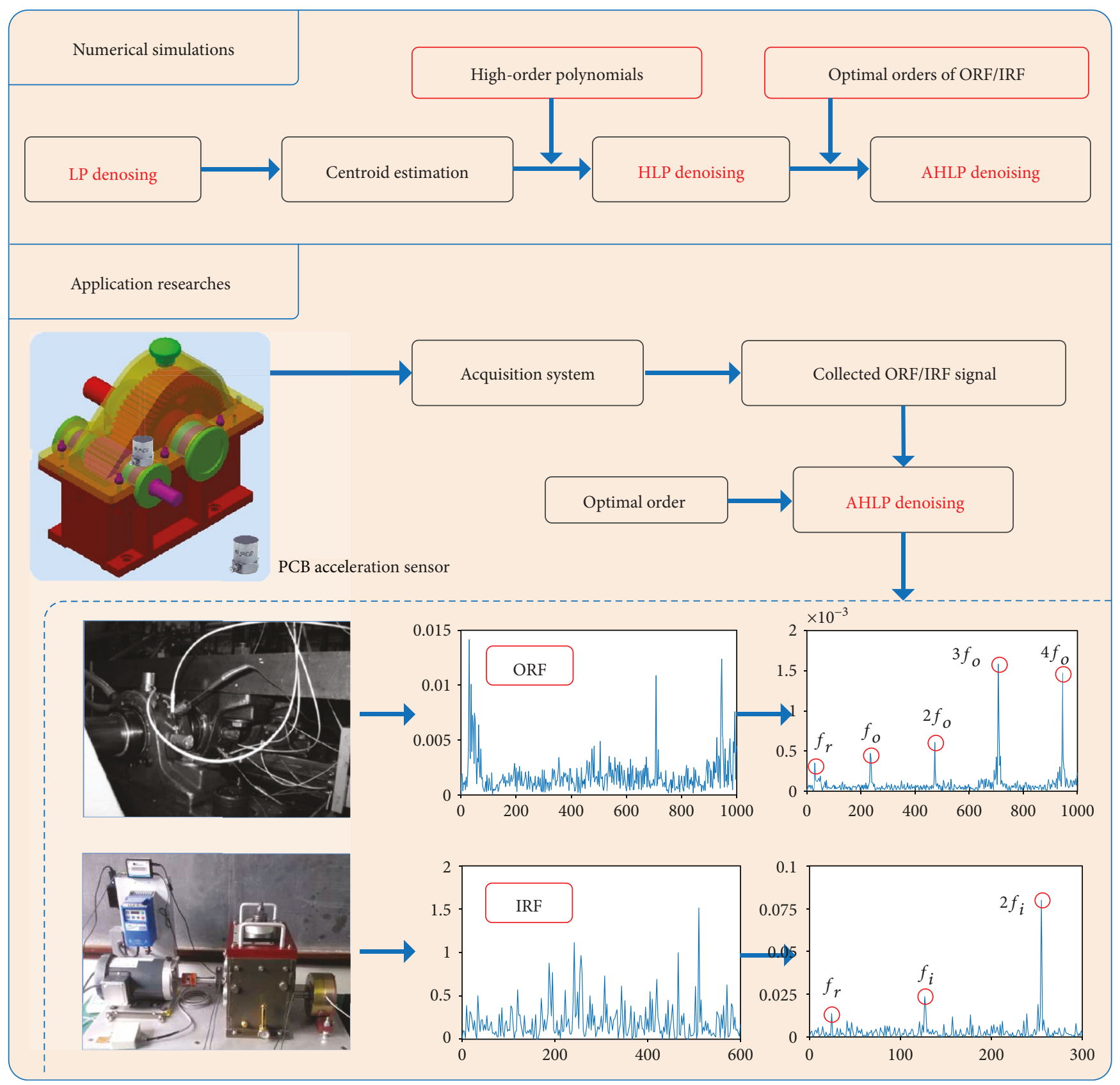

FIGURE 2: The scheme of AHLP denoising method in fault diagnosis of rolling bearing.

was proposed by using mean of phase points of neighborhood as centroid of neighborhood [34, 35].

$$
\overline{\mathbf{X}}_{n}=\frac{1}{U_{n}} \sum_{k \in U_{n}} \mathbf{X}_{k}
$$

This method may generate certain errors because of local linearization; the centroids of each neighborhood are secants instead of tangents and all centroids (blue circles) are shifted inward regarding the curvature, as shown in Figure 1(a). Afterwards, a method of estimating the centroid by using the second-order polynomial was developed to further suppress noise [29].

$$
\overline{\mathbf{X}}_{n}^{\prime}=2 \overline{\mathbf{X}}_{n}-\frac{1}{U_{n}} \sum_{k \in U_{n}} \overline{\mathbf{X}}_{k}
$$

where a tangent approximation is obtained by shifting the centroid of neighborhood outward regarding the curvature. The illustration is shown in Figure 1(b), where the filled circle denotes the centroid of phase points of adjacent neighborhoods, and the square is the modified centroid. 
TABLE 3: The parameters of the vibration signal model of ORF rolling bearing.

\begin{tabular}{ccccccccc}
\hline$A_{0}$ & $f_{m}$ & $f_{o}$ & $\tau_{i}$ & $f_{n}$ & $C_{A}$ & $\varphi_{A}$ & $\varphi_{w}$ & $B$ \\
\hline 3 & 0 & 100 & 0.01 & 2000 & 1 & 0 & 0 & 800 \\
\hline
\end{tabular}

Essentially, the method is performed by moving the centroid outward to make it approximately tangent to the hyperplane of projection. The above two methods are referred here as standard LP denoising method.

2.2. High-Order Local Projection Method. The method was proposed by applying high-order polynomials to estimate the centroid of the neighborhood more accurately. Significant achievements have been obtained by high-order algorithms in medical signal processing [36] and thermodynamics [37], because it can reflect mathematical characteristics more accurately. In this paper, this method is referred as the HLP denoising method. The basic principle is explained below.

As for any real number $\delta>0$, define a continuous moving average operator as $I_{\delta}$.

$$
\left(I_{\delta} f\right)(x)=\frac{1}{2 \delta} \int_{x-\delta}^{x+\delta} f(t) d t
$$

where $f(t)$ is a time series, and $\delta$ is shift step length.

The effect of the LP denoising method is enhanced by considering using the linear combination of the first and the second orders to estimate the centroid of neighborhood, thus improving the denoising effect. Hence, it can be inferred that

$$
f_{n}(0)=a_{1}\left(I_{\delta} f_{n}\right)(0)+a_{2}\left(I_{\delta}^{2} f_{n}\right)(0)
$$

where $f_{n}=(0)$ is applicable to all monomials, when $n=0$, $f_{n}(x)=1$, and when $n \neq 0, f_{n}(x)=x^{n}, 0 \leq n \leq n_{\text {max }}$, while $n_{\max }$ is supposed to be as large as possible. As for any odd number, both sides of (5) are 0 . Setting $n=0$ and $n=2$ results in a system combined with two linear equations, which have unique solution $a_{1}=2$ and $a_{2}=-1$. In the $m$ dimensional Euclidean space, the centroid of all phase points within a ball of radius $\delta$ is equivalent of moving average operator $I_{\delta}$. In this way, the second-order LP denoising method was proposed. If (5) is applicable to all monomials, $f_{n}(x)=x^{n}, 0 \leq n \leq 3$, which means

$$
p_{3}(x)=a_{1}\left(I_{\delta} p_{3}\right)(x)+a_{2}\left(I_{\delta}^{2} p_{3}\right)(x)
$$

where $p_{3}(x)$ holds accurately for the third-order Taylor polynomial of any thrice differentiable function. As for $p_{3}(x)$ in (6), which is analogue of $f_{n}(0)$ in (5), it holds exactly for multivariate Taylor polynomials of higher order up to three in each variable.

Then for the HLP denoising method, the linear combination of $I_{\delta}^{i}$ needs to be identified to represent higher-order
Taylor polynomial more accurately. Through induction and deduction, it can be obtained that as for each $j, n \in N$,

$$
\begin{aligned}
\left(I_{\delta}{ }^{j} f_{n}\right)(x)= & \frac{1}{\delta^{n+j}}\left(-\frac{1}{2}\right)^{j} \frac{n !}{(n+j) !} \\
& \times \sum_{r=0}^{j}\left(\begin{array}{c}
j \\
r
\end{array}\right)(-1)^{r}(x+(2 r-j) \delta)^{n+j}
\end{aligned}
$$

This formula is used to prove that as for $k=1,2, \ldots, 20$, the system constituted by $k$ linear equations has the unique solution. The system and the solution are as follows:

$$
\begin{aligned}
f_{n}(0) & =\sum_{i=1}^{k} a_{i}\left(I_{\delta}^{i} f_{n}\right)(0), \quad n=0,2, \ldots, 2(k-1), \\
a_{i} & =(-1)^{i-1}\left(\begin{array}{l}
k \\
i
\end{array}\right), \quad i=1,2, \ldots, k .
\end{aligned}
$$

Obviously, all coefficients determined by (9) include two situations of estimating centroid of neighborhood when order is 1 and 2, namely, $i=1$ [34] and $i=2$ [29]. The equation also expands the estimation of centroid of neighborhood to higher orders $(i \geq 3)$ such as $i=1,2, \ldots, k$. The difference between algorithms of standard LP and HLP denoising methods lies in the 3rd step of all procedures as shown in Table 1. The 3rd step of the standard LP denoising method uses the mean and second-order polynomial to calculate the centroid of the local neighborhood. As for HLP denoising method, higher-order polynomials are utilized to estimate the centroid.

In this paper, the orders up to 8 are analyzed. In mathematics, binomial coefficients refer to a set of positive integers appear in binomial theorem as coefficients. They are indexed by two nonnegative integers, namely, $k$ and $i$, which are the coefficients of $x^{i}$ term in the polynomial expansion of binomial power $(1+x)^{k}$. Under appropriate situations, the value of the coefficient $\left(\begin{array}{l}k \\ i\end{array}\right)$ is set by $(k !) / i !(k-i)$ !. Arranging binomial coefficients into rows for $i$ changes from 1 to $k$. Thus, during the algorithm, the solutions $a_{i}$ of different orders can be derived and calculated as given in Table 2 . The left side shows the coefficients of deployed high polynomials.

\subsection{Adaptive High-Order Local Projection Denoising Method.} As mentioned above, as a denoising reduction method, LP method has been verified to be effective in reducing noises existing in vibration signals of rolling bearings after theoretical derivation and extensive tests. Estimation of the centroid of the neighborhood by using high-order polynomials is beneficial to achieve better denoising effect; hence, HLP denoising method is adopted in this paper to deal with vibration signals of faulty rolling bearings. Owing to the reason that vibration signals of ORF and IRF rolling bearings have different nonlinear geometric structures, denoising effects of different orders differ, so the optimal orders of different kinds 


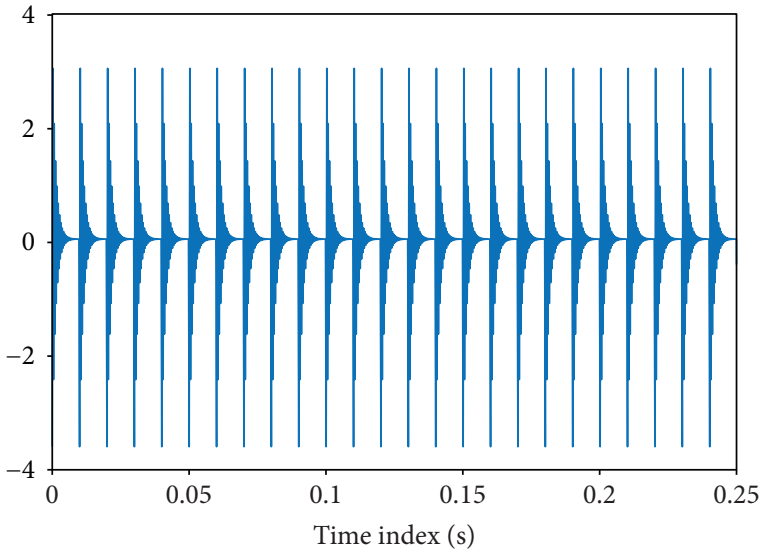

(a)

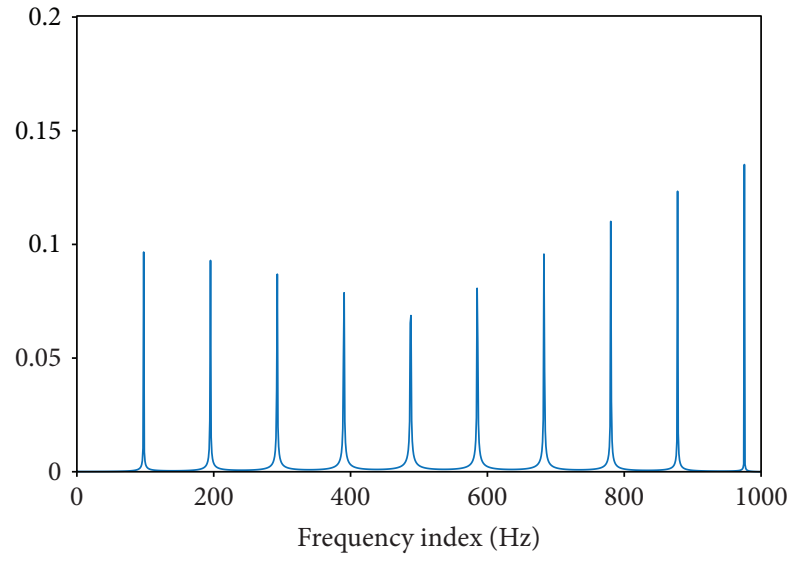

(b)

FIGURE 3: Time and frequency domain plots of simulated vibration signal of ORF rolling bearing.

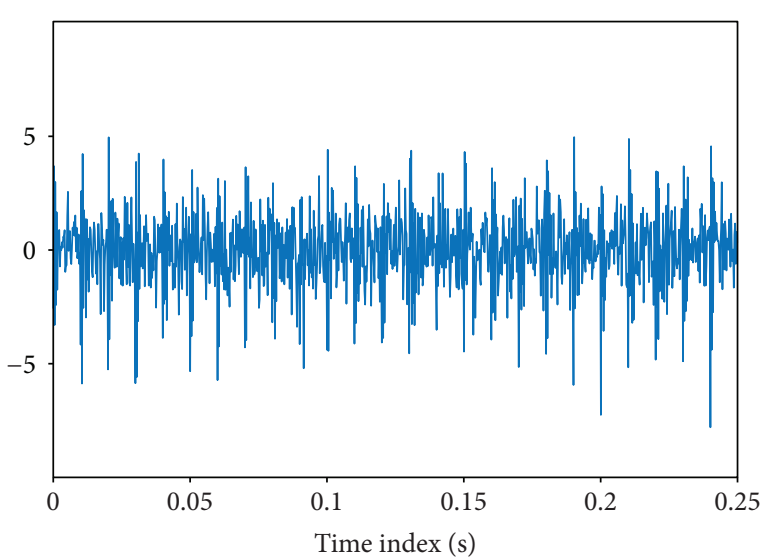

(a)

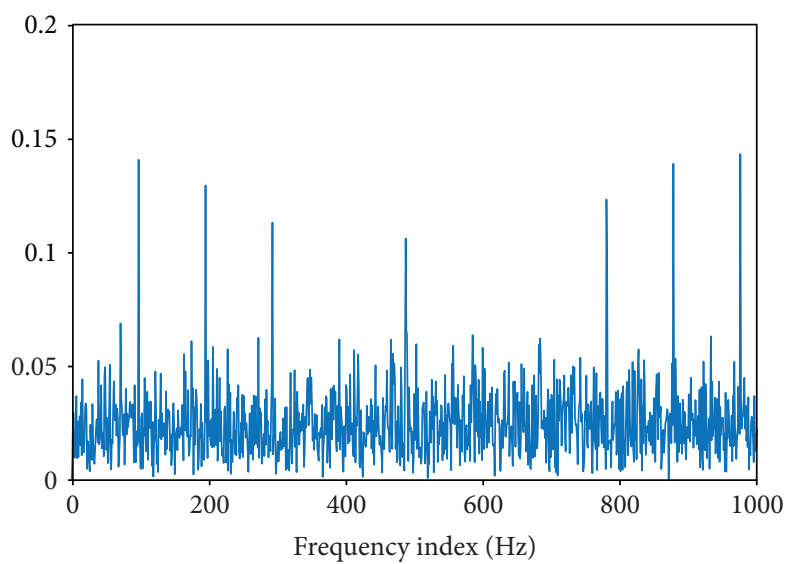

(b)

FIGURE 4: Time and frequency domain plots of simulated noisy signal of ORF rolling bearing.

TABLE 4: The SNRs of denoised signal with different orders.

\begin{tabular}{lcccccccc}
\hline Order & 1 & 2 & 3 & 4 & 5 & 6 & 7 & 8 \\
SNR & 8.92 & 9.73 & 10.21 & $\mathbf{1 1 . 6 2}$ & 8.50 & 5.61 & 5.09 & 4.43 \\
\hline
\end{tabular}

of faults are different. By choosing the optimal orders before conducting denoising process, a novel AHLP denoising is hereby proposed in this paper, and the scheme of the proposed method is illustrated in Figure 2.

\section{Numerical Simulations}

3.1. Vibration Signal Model of Faulty Rolling Bearing. The vibration signal model of faulty rolling bearing used hereinafter was proposed by Randall et al. [40-42], and it takes rolling bearing's construction (geometry), tolerance, amplitude modulation, ball sliding, surface wear, and other factors into consideration. This bearing model has been successfully applied in related researches [42-44]. The main faults of rolling bearing include local pitting on the inner ring, outer ring, and rolling element, which are caused by shock effects on the rolling bearing and other parts during the operation of the entire system. The frequency of the periodic impact reflects the type of rolling bearing faults. A local pitting often encourages the system to produce vibration of the inherent frequency of the rolling bearing or the system. During the operation of the rolling bearing, the absolute position of pitting may change periodically versus sensor position. For instance, when a local pitting occurs on the inner ring, the absolute position of the local pitting would change with the rotation speed periodically, and when it occurs on the rolling element, the absolute position would change with the rotation period of bearing retainer periodically. Since the rotation period of the absolute position of a local pitting is greater than the period of shock, the shock is modulated distinctively. Compared to the derivative of resonant frequency, the shock time is rather short and usually decays rapidly. Under a steady-state condition, it can be considered that modulation amplitude does not change during the process 


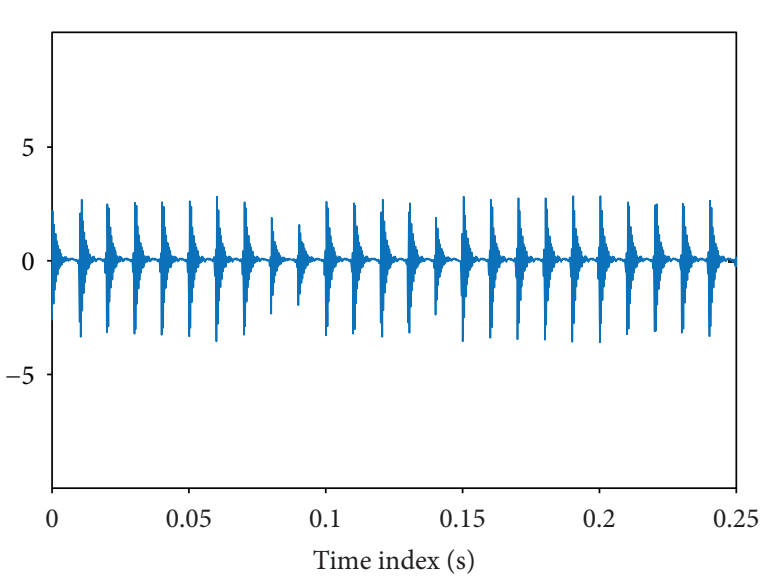

(a)

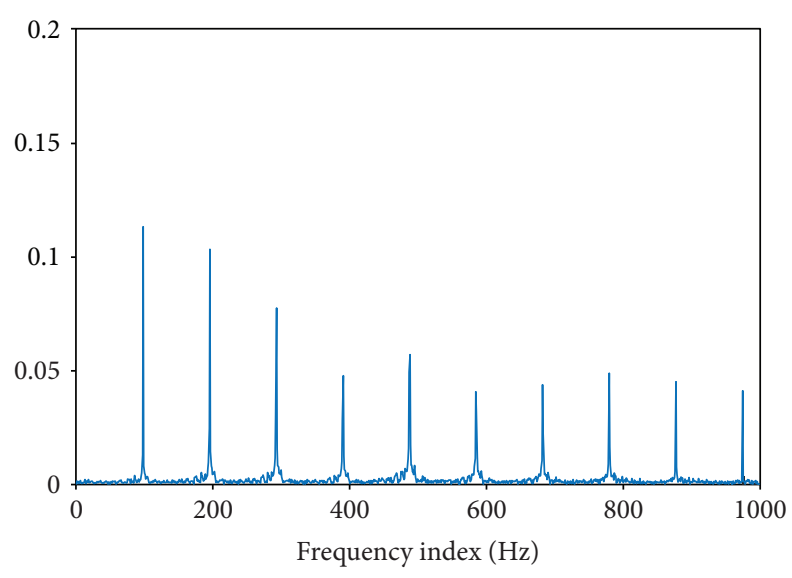

(b)

FIGURE 5: Time and frequency domain plots of denoised signal of ORF rolling bearing.

TABLE 5: The SNRs of denoised signal with different orders.

\begin{tabular}{|c|c|c|c|c|c|c|c|c|c|}
\hline Order & & 1 & 2 & 3 & 4 & 5 & 6 & 7 & 8 \\
\hline \multirow{5}{*}{ SNR } & 1 & 92 & J & .21 & 1. & .50 & 5.61 & 5.09 & 4.43 \\
\hline & 2 & .17 & 42 & 15.65 & 5.63 & 12.46 & 10.30 & 8.89 & 7.06 \\
\hline & 3 & 11.65 & 15 & 14.90 & 16 & 14.70 & 13.97 & 10.45 & 7.90 \\
\hline & 4 & 12.7 & & 18 & 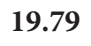 & 0 & 13 & 12.38 & 10.66 \\
\hline & 5 & 12.83 & 13.78 & 14.67 & 17.01 & 14.38 & 13.78 & 11.79 & 11.51 \\
\hline
\end{tabular}

of shock oscillation, and the oscillation signals caused by each shock are the same.

Define $T$ as the period of shock and $s(t)$ as the shock oscillation caused by a pitting fault. Assume that the amplitude of $i$ th shock is $A_{i}$. There are generally strong ambient noises since the working environment of rolling bearing is relatively poor. Thus, the model considers the interference of additive noises $n(t)$, which are assumed to be stationary random noises of zero mean. Hence, the vibration signal model of faulty rolling bearing $m(t)$ is expressed as follows:

$$
\begin{aligned}
m(t) & =\sum_{i=1}^{M} A_{i} \cdot s\left(t-i T-\tau_{i}\right)+n(t), \\
A_{i} & =A_{0} \cdot \cos \left(2 \pi f_{m} t+\varphi_{A}\right)+C_{A}, \\
s(t) & =\exp (-B t) \cdot \cos \left(2 \pi f_{n} t+\varphi_{w}\right),
\end{aligned}
$$

where $A_{0}$ is amplitude modulation factor function, $f_{m}$ is modulation frequency, $s(t)$ is shock oscillation, $\tau_{i}$ is tiny slippage between rolling element and raceway, $f_{n}$ is system resonant frequency of rolling bearing, $\varphi_{A}, \varphi_{w}$ and $C_{A}$ are arbitrary normal numbers, and $B$ is the intensity of resonance. The significant distinction between vibration signals of simulated ORF and IRF rolling bearings is when it comes to ORF rolling bearing that $f_{m}$ equals to $0 \mathrm{~Hz}$, while when it comes to IRF rolling bearing that $f_{m}$ equals to $f_{r}\left(f_{r}\right.$ denotes rotation frequency).

3.2. Numerical Simulation of ORF Rolling Bearing. Based on the vibration signal model of faulty rolling bearing in Section
TABLE 6: The parameters of the vibration signal model of IRF rolling bearing.

\begin{tabular}{ccccccccc}
\hline$A_{0}$ & $f_{m}$ & $f_{i}$ & $\tau_{i}$ & $f_{n}$ & $C_{A}$ & $\varphi_{A}$ & $\varphi_{w}$ & $B$ \\
\hline 3 & 20 & 100 & 0.01 & 2000 & 1 & 0 & 0 & 800 \\
\hline
\end{tabular}

3.1, as for the ORF rolling bearing, $f_{m}$ was set as $0 \mathrm{~Hz}$ in our research; $f_{o}$ denotes the fault frequency of ORF rolling bearing. The parameters of the simulated vibration signal of ORF rolling bearing are set as shown in Table 3 in this paper. The sampling point is $N=4096$ and the sampling frequency is $f_{s}=4096 \mathrm{~Hz}$. To verify the effectiveness of HLP denoising method in real situations, where the collected signal normally has noise, therefore, Gaussian white noise is added in numerical simulations. The power spectral density of Gaussian white noise is uniformly distributed, and its amplitudes obey the Gaussian distribution, making it is suitable for simulation analysis. Here, Gaussian white noise is added to the original signal, and the signal noise ratio (SNR) is 1 . The time and frequency domain plots of the simulated vibration signal of ORF rolling bearing are shown in Figure 3. The time and frequency domain plots of the simulated noisy signal of ORF rolling bearing are shown in Figure 4. The time domain of the first $0.25 \mathrm{~s}$ of the signal is analyzed specifically to show the effectiveness of the HLP denoising method and clarify different characteristics between vibration signals of ORF and IRF rolling bearings.

It can be observed from the time and frequency domain plots in Figure 3 that the vibration signal of ORF rolling bearing has periodic characteristic. The frequency domain plot shows fault frequency $f_{o}=100 \mathrm{~Hz}$ and its harmonic frequencies containing second, third, and fourth harmonic frequency. By conducting HLP denoising method towards simulated noisy ORF signal with different orders, the denoised signal with different SNRs can be obtained as shown in Table 4.

It can be seen from Table 4 that when the original SNR is 1 , the SNRs of the denoised signal with different orders increase along with the order until 4 and then decrease all 


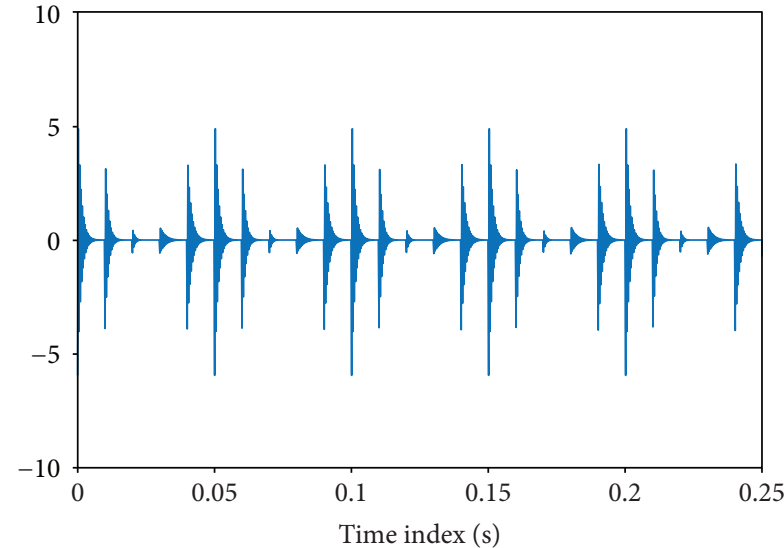

(a)

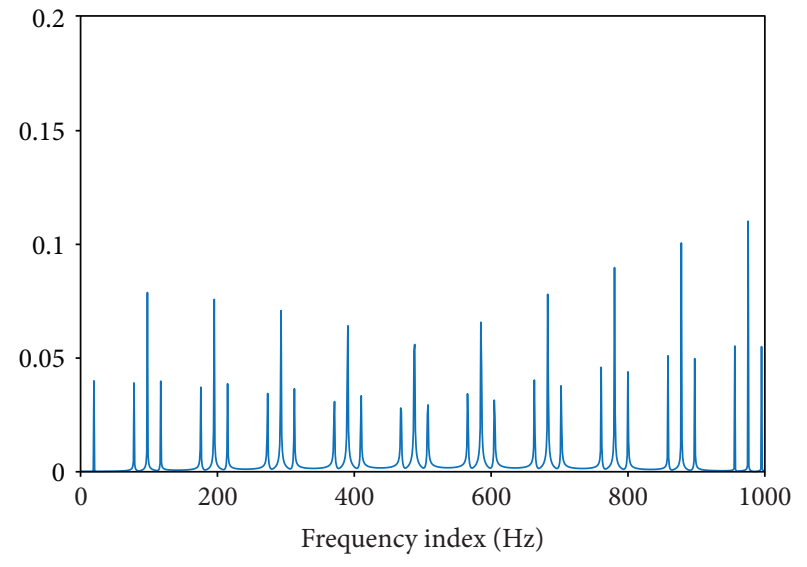

(b)

FIGURE 6: Time and frequency domain plots of simulated vibration signal of IRF rolling bearing.

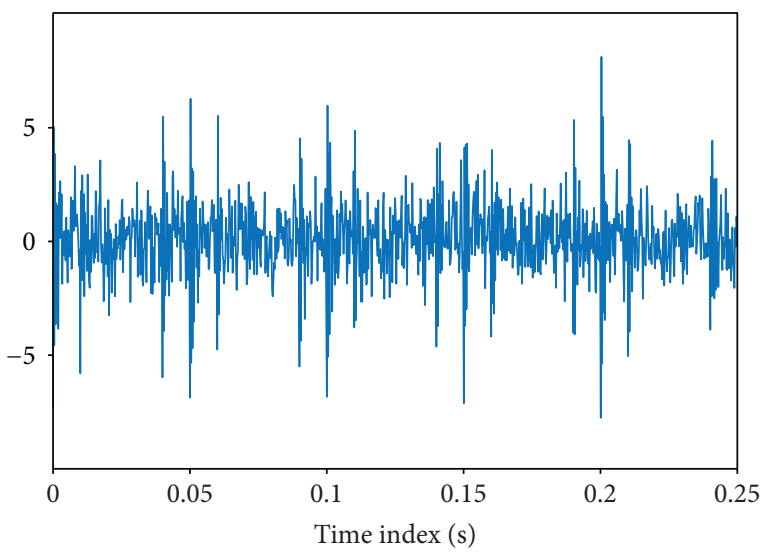

(a)

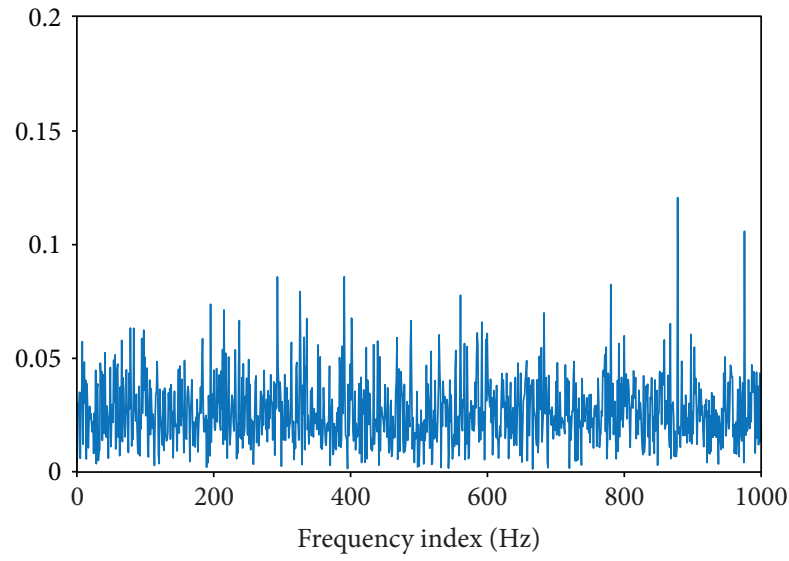

(b)

FIGURE 7: Time and frequency domain plots of simulated noisy signal of IRF rolling bearing.

TABLE 7: The SNRs of denoised signal with different orders.

\begin{tabular}{lcccccccc}
\hline Order & 1 & 2 & 3 & 4 & 5 & 6 & 7 & 8 \\
SNR & 6.91 & 7.28 & 7.73 & 7.62 & 7.20 & 6.72 & 5.07 & 4.52 \\
\hline
\end{tabular}

the way. The time and frequency domain plots of the denoised vibration signal of ORF rolling bearing, when the order of HLP denoising method is 4, are shown in Figure 5.

As shown in Figure 5, the characteristic frequencies $f_{o}$ and its resonant frequencies can be clearly extracted under the influence of noise. The characteristic frequency amplitudes are obvious. The result verifies the effectiveness of HLP denoising method to extract the fault frequencies of vibration signal of ORF rolling bearing. To obtain the optimal orders for HLP denoising method aiming at vibration signal of ORF rolling bearing, Gaussian white noise with different SNRs, $\mathrm{SNR}=1,2,3,4,5$, respectively, is added to the original simulated ORF signal. Then, different SNRs of different denoised results are obtained, as shown in Table 5.

When the SNR is bigger than 5, which implies there are not too much additive noises in the signal, the HLP can achieve proper denoising effect in most cases. Hence, the above simulations are conducted to find optimal orders aiming at vibration signal of ORF rolling bearing. As shown in Table 5 , the primary optimal order of HLP denoising method is 4 . In one case, the optimal order is 3 , and SNR is only a little bit higher than the situation when the order is 4 . Because of some uncertain parameters in vibration signal model of faulty rolling bearing, the slight fluctuation of optimal order can be accepted. Therefore, it can be concluded that the optimal order for vibration signal of ORF rolling bearing is 4 .

3.3. Numerical Simulation of IRF Rolling Bearing. Based on the vibration signal model of faulty rolling bearing in Section 3.1 , as for the IRF rolling bearing, $f_{m}$ and $f_{r}$ were set as $20 \mathrm{~Hz}$ in our research; $f_{i}$ denotes the fault frequency of IRF rolling bearing. The parameters of the simulated vibration signal of IRF rolling bearing are set as shown in Table 6 in this paper. The sampling point is $N=2048$ and the sampling frequency is $f_{s}=4096 \mathrm{~Hz}$. Similar to that in Section 4.1, Gaussian white noise is added to the original signal, and the SNR is 1 . The time and frequency domain plots of the simulated vibration signal of IRF rolling bearing are shown in Figure 6. The time 


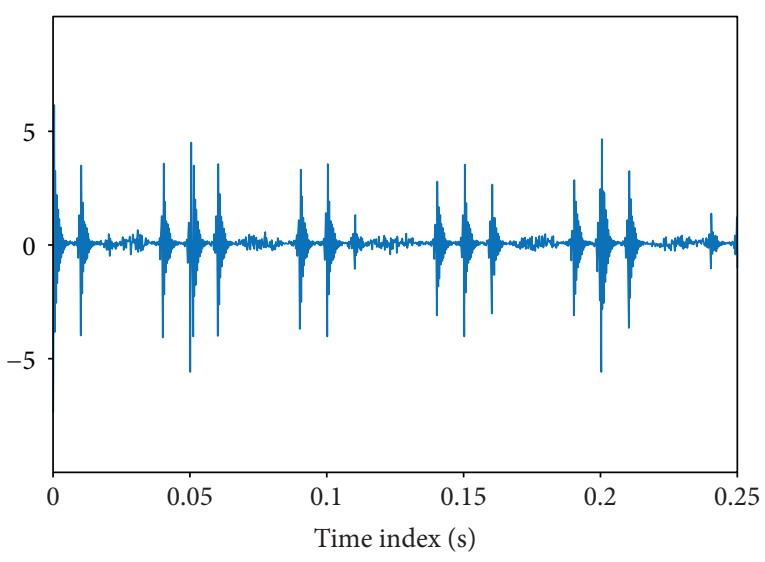

(a)

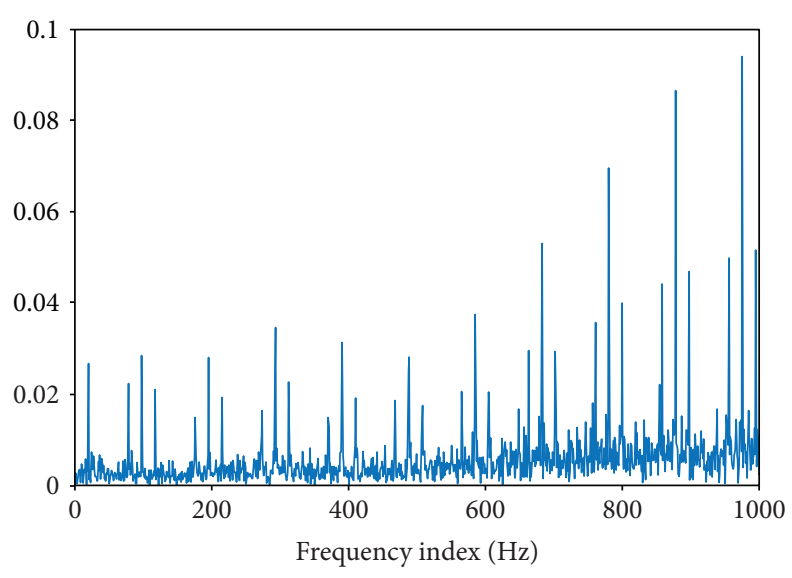

(b)

FIgURE 8: Time and frequency domain plots of the denoised signal of IRF rolling bearing.

TABLE 8: The SNRs of denoised signal with different orders.

\begin{tabular}{|c|c|c|c|c|c|c|c|c|c|}
\hline Order & & 1 & 2 & 3 & 4 & 5 & 6 & 7 & 8 \\
\hline \multirow{5}{*}{ SNR } & 1 & 6.91 & 7.28 & 7.73 & 7.62 & 7.20 & 6.72 & 5.07 & 4.52 \\
\hline & 2 & 7.68 & 8.04 & 8.54 & 8.30 & 6.62 & 6.61 & 5.93 & 5.12 \\
\hline & 3 & 8.14 & 8.25 & 8.66 & 8.41 & 8.12 & 6.55 & 6.53 & 5.89 \\
\hline & 4 & 8.73 & 8.81 & 9.03 & 8.70 & 8.44 & 7.50 & 7.17 & 6.81 \\
\hline & 5 & 8.82 & 9.25 & 9.32 & 8.59 & 8.15 & 8.06 & 7.73 & 7.01 \\
\hline
\end{tabular}

and frequency domain plots of the simulated IRF noisy signal are shown in Figure 7 . The time domain of the first $0.25 \mathrm{~s}$ of the signal is analyzed specifically to show the effectiveness of HLP denoising method and clarify different characteristics between vibration signals of ORF and IRF rolling bearings.

It can be observed from the time and frequency domain plots in Figure 6 that the vibration signal of IRF rolling bearing has periodic characteristic. The frequency domain plot shows fault frequency $f_{i}=100 \mathrm{~Hz}$ and its harmonic frequencies containing the second, third, and fourth harmonic frequency. In addition to characteristic frequencies, there are sideband frequencies with modulation frequency $f_{m}=f_{r}=$ $20 \mathrm{~Hz}$ and the resonant frequencies are $f_{i} \pm f_{r}, 2 f_{i} \pm f_{r}, 3$ $f_{i} \pm f_{r}, \ldots$ and $f_{r}=20 \mathrm{~Hz}$ in the low-frequency range. Apply the HLP denoising method towards the noisy IRF signal with different orders that result in the denoised signals with different SNRs, as shown in Table 7.

It is clear from Table 7 that, when the original SNR is 1 , the SNRs of the denoised signal with different orders increase with the order until 3 and then decrease all the way. The time and frequency domain plots of the denoised ORF noisy signal, when order of HLP denoising method is 3, are shown in Figure 8.

As shown in Figure 8, the characteristic frequencies $f_{0}$ and its resonant frequencies $f_{i} \pm f_{r}, 2 f_{i} \pm f_{r}, 3 f_{i} \pm f_{r}, \ldots$ and $f_{r}=20 \mathrm{~Hz}$ can be clearly extracted under the influence of noise. The characteristic frequency amplitudes are obvious. The result demonstrates the effectiveness of HLP denoising method in extracting fault frequencies of the vibration signal of IRF rolling bearing. To obtain the optimal orders for HLP denoising method aiming at vibration signal of IRF rolling bearing, Gaussian white noise with different SNRs, $\mathrm{SNR}=1,2,3,4,5$, respectively, is added into original simulated IRF signal. Then, different SNRs of different denoised results are obtained, as shown in Table 8 .

As shown in Table 8, the optimal order of the HLP denoising method is 3 . Different from the situation when HLP denoising method is applied to ORF signal, the optimal order is a constant. Therefore, it can be obtained that the optimal order for HLP denoising method aiming at the vibration signal of IRF rolling bearing is 3 .

\section{Applications to Fault Diagnosis of ORF and IRF Rolling Bearings}

Through numerical simulations, the proposed novel AHLP denoising method is applied to deal with vibration signals of ORF and IRF rolling bearings successfully, and there are certain optimal orders for different faults of rolling bearing. By choosing optimal order of HLP, the best denoising effect can be obtained. Here, two cases of test data are used to validate the effectiveness of the proposed AHLP denoising method in this paper.

4.1. Application to Processing of Intelligent Maintenance System Bearing Data. To verify the effectiveness of the proposed method in application to fault diagnosis of rolling bearing, the bearing data from Intelligent Maintenance Systems (IMS) Center of University of Cincinnati [45] is used to verify the proposed method. Three datasets are included in the downloaded data packet, and a test to failure experiment was conducted and dataset was collected by NI DAQ Card 6062E during no. 2 experiment. The 772nd data of no. 2 dataset was used to verify the effectiveness of the proposed method in this paper. The schematic diagram of the experimental apparatus and the position of sensors are shown in Figure 9. Four Rexnord ZA-2115 rolling bearings were installed on the shaft, and PCB 353B33 accelerometers were installed vertically on the bearing houses. The rotational speed was $2000 \mathrm{r} / \mathrm{min}$, facilitated by an AC motor, namely, 


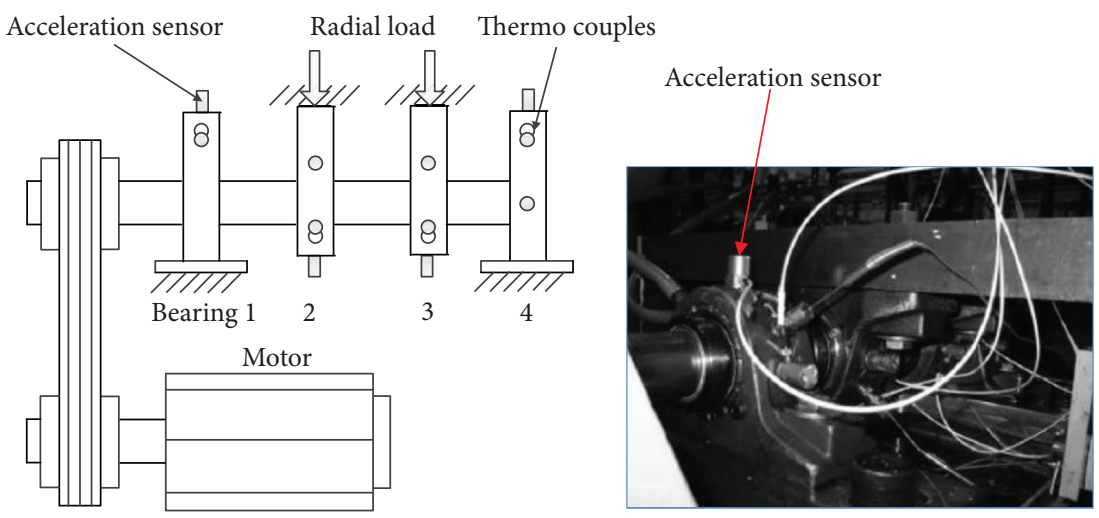

FIgURE 9: The schematic diagram and a picture of the apparatus [45].

TABLE 9: Rolling element bearing parameters.

Rolling element bearing parameters of ZA-2115 (diameter/cm)

\begin{tabular}{lccc}
\hline Ball number $n$ & Contact angle $\alpha$ & Ball diameter $d_{r}$ & Pitch diameter $D_{\mathrm{w}}$ \\
16 & 15.17 & 0.331 & 2.815 \\
\hline
\end{tabular}

TABLE 10: Bearing characteristic frequencies.

\begin{tabular}{lc}
\hline Fault type & Failure frequency \\
\hline Defect on inner race & $f_{i}=0.5 n\left(1+d_{r} \cos \alpha / D_{w}\right) f_{r}$ \\
Defect on outer race & $f_{o}=0.5 n\left(1-d_{r} \cos \alpha / D_{w}\right) f_{r}$ \\
\hline
\end{tabular}

the rotational frequency $f_{r}$ is $33.33 \mathrm{~Hz}$. The sampling frequency is $20 \mathrm{kHz}$. The specific parameters of fault rolling bearings are shown in Table 9. The calculating methods for different rolling bearing fault frequencies are shown in Table 10. The characteristic frequencies of rolling element bearing were computed as shown in Table 11.

At the end of the no. 2 experiment, ORF happened on the rolling bearing. The time and frequency domain plots of the collected vibration signal of the faulty rolling bearing (772nd data) are shown in Figure 10.

The optimal order is chosen as 4 among the proposed AHLP denoising method. To illustrate the effectiveness of the proposed method, the frequency domain plots of the original signal and signal processed by WPD are also presented. The wavelet function db15 with 11 layers is adopted in the WPD. To observe the fault characteristic frequency clearly and conduct a comparative analysis to verify the effectiveness of the proposed method, the partial enlarged frequency plots of frequency domain of collected signal and WPD denoised signal are shown in Figure 11. The frequency domain plots of signal processed by the LP denoising method and the proposed AHLP denoising method are presented in Figure 12.

It can be seen from Figure 11(a) that in the frequency domain plot of collected signal of ORF rolling bearing, the rotational frequency $f_{r}$ can be seen along with harmonic frequencies $3 f_{o}$ and $4 f_{o}$, but the fault characteristic frequency itself $f_{o}$ cannot be found. It indicates that the rolling bearing
TABle 11: Characteristic frequencies of Rexnord ZA-2115 rolling bearing.

\begin{tabular}{lc}
\hline Fault type & Fault frequency $(\mathrm{Hz})$ \\
\hline Outer ring fault & $f_{o}=236.4$ \\
Inner ring fault & $f_{i}=296.9$ \\
\hline
\end{tabular}

had severe fault then and collected signal was interfered by background noises to a great extent, and there are many interfering frequencies. From Figure 11(b) and Figure 12(a), it can be seen after the processing of WPD and LP denoising method that all characteristic frequencies can be extracted but still along with some interfering frequencies. In addition, the effectiveness of LP denoising method is better than WPD. It can be seen from Figure 12(b) that after AHLP denoising method, the background noise is greatly reduced and eliminated, the characteristic frequencies are obvious, and there are not many interfering frequencies in the frequency domain plot. The frequency domain plot shows very clear the rotational frequency $f_{r}$, and fault characteristic frequencies $f_{o}, 2 f_{o}, 3 f_{o}, 4 f_{o}$, namely, fault characteristic frequencies can be well extracted. The denoising effect of AHLP is better than LP and WPD denoising methods, which indicates that the extension research from LP to AHLP is a successful exploration. Based on the displayed results and comparative analysis, it can be concluded that the vibration signal of ORF rolling bearing with severe fault can be well denoised by the proposed AHLP denoising method in practical application.

4.2. The Collected Signal Processing of Drivetrain Diagnostics Simulator. To further verify the effectiveness of the proposed method in the application of weak fault diagnosis, the experiment is conducted on Drivetrain Diagnostics Simulator. Here, ORF and IRF rolling bearings are used during the experiment, and fault signals are collected to be processed. 


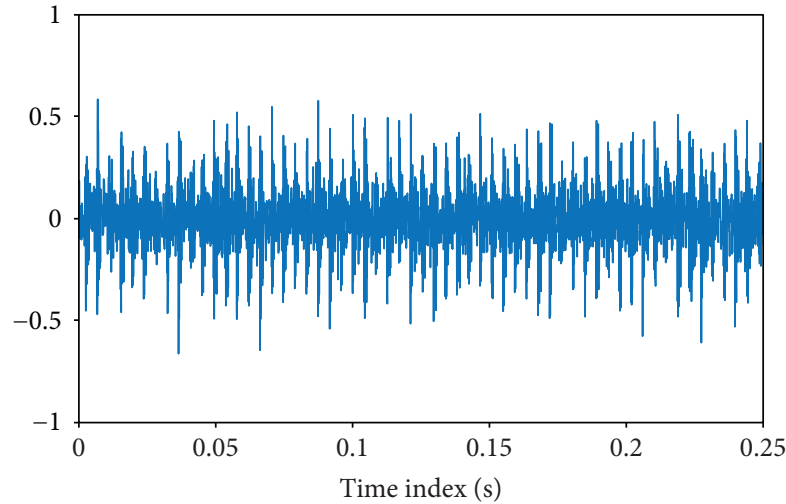

(a)

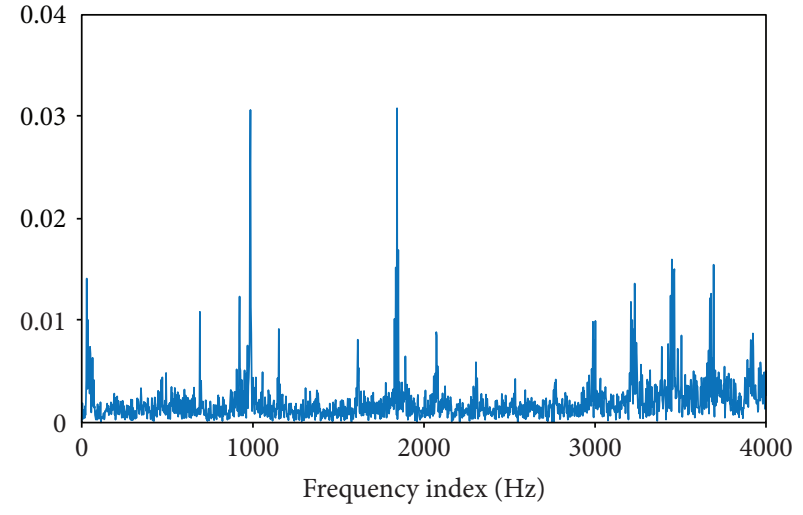

(b)

FIGURE 10: Time and frequency domain plots of collected vibration signal of ORF rolling bearing.

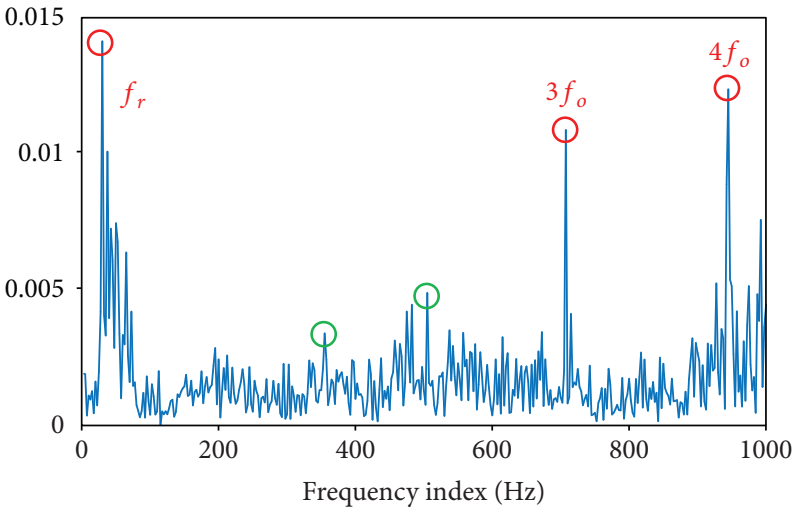

(a)

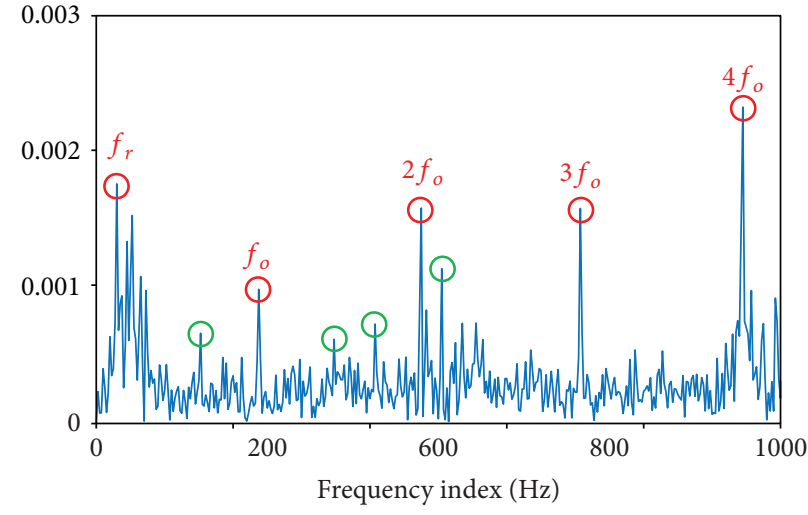

(b)

FIGURE 11: (a) Frequency domain plot of collected signal. (b) Frequency domain plot of denoised signal by WPD (red circles denote characteristic frequency, and green circles denote interfering frequency, as same hereinafter).

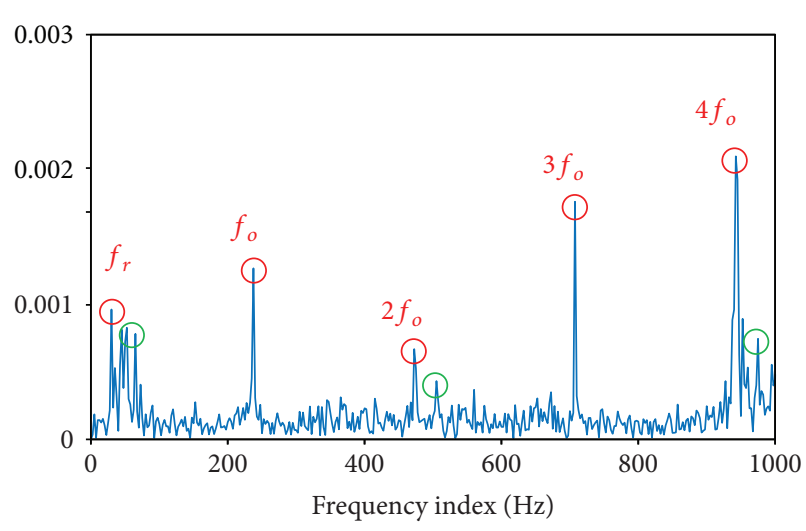

(a)

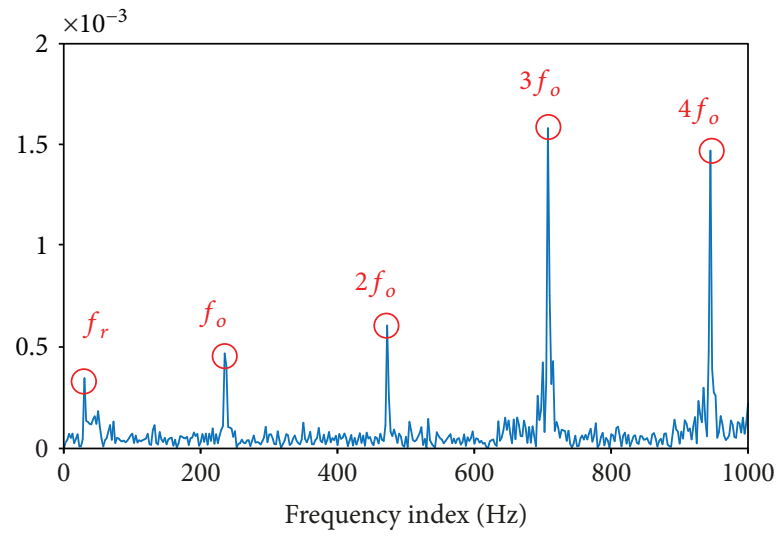

(b)

FIGURE 12: (a) Frequency domain plot of denoised signal by LP denoising method. (b) Frequency domain plot of denoised signal by AHLP denoising method.

The experimental apparatus is produced by SQI Company, United States. The experiment is aimed at collecting the signal of ORF and IRF rolling bearings. The sensor is placed on the rolling bearing end plate to collect its acceleration signal.
The experimental apparatus is composed of a variable speed drive, a torque transducer and encoder, a parallel shaft gearbox which includes two parallel shaft rolling bearing, and a programmable magnetic brake. The schematic diagram of 


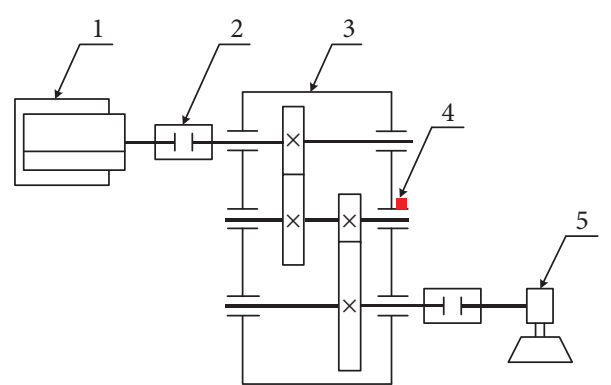

- Locations of sensor

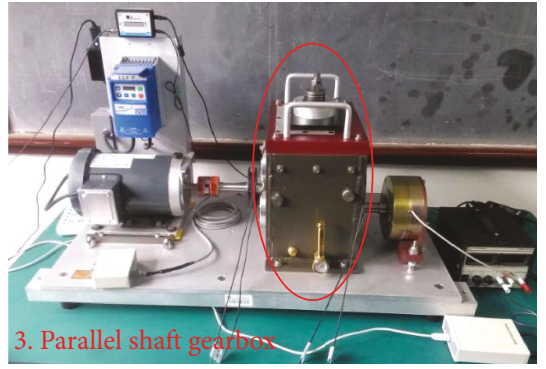

(b)

FIGURE 13: The schematic diagram and the picture of the experimental apparatus. 1: variable speed drive, 2: torque transducer and encoder, 3: parallel shaft gearbox, 4: test point, and 5: programmable magnetic brake.

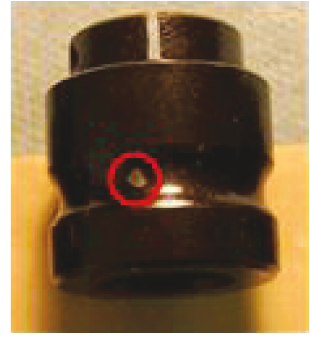

(a)

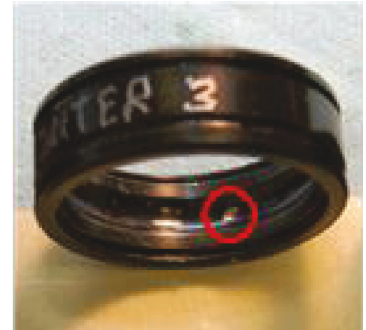

(b)

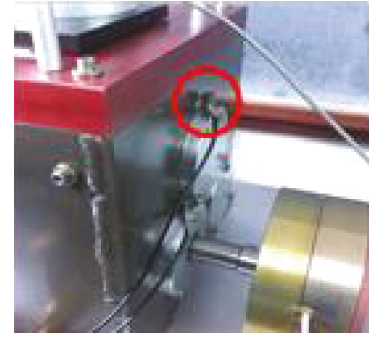

(c)

FIGURE 14: (a) IRF of rolling bearing, (b) ORF of rolling bearings, and (c) location of sensor.

TABLE 12: Characteristic frequencies of FAFNIR deep groove rolling bearing.

\begin{tabular}{lc}
\hline Fault type & Fault frequency $(\mathrm{Hz})$ \\
\hline Inner ring fault & $f_{i}=130.32$ \\
Outer ring fault & $f_{o}=85.73$ \\
\hline
\end{tabular}

the experimental apparatus and its photo are shown in Figure 13. The photos of IRF and ORF rolling bearings and location of sensor are shown in Figure 14.

The experiment was conducted to collect the vibration signal of weak fault rolling bearing. During the experiment, the sampling frequency is $8192 \mathrm{~Hz}$, and the rotational frequency $f_{r}$ is $25 \mathrm{~Hz}$. The fault characteristic frequencies of experimental FAFNIR deep groove rolling bearing are shown in Table 12.

The time and frequency domain plots of the collected signal of fault rolling bearing are shown in Figure 15.

The vibration signal of IRF rolling bearing is used here to verify the effectiveness of proposed AHLP denoising method; thus, the optimal order can be chosen as 3 among the proposed AHLP denoising methods. To better analyze the denoising effect, the frequency range of $0-300 \mathrm{~Hz}$ is analyzed. To observe the fault characteristic frequency clearly and conduct a comparative analysis to verify the effectiveness of the proposed method, the partially enlarged frequency plots of frequency domain of collected signal and WPD denoised signal are shown in Figure 16. The frequency domain plots of the vibration signal processed by the LP denoising method and the proposed AHLP denoising method are presented in Figure 17.

It can be seen from Figure 16(a) that in the frequency domain plot of collected signal of IRF rolling bearing, the collected signal was interfered by background noises to a great extent, and there are many interfering frequencies. The rotational frequency and fault characteristic frequencies cannot be found, and it indicates there was an incipient fault happened on rolling bearing. From Figure 16(b), it can be seen after the processing of WPD that the harmonic frequency 2 $f_{i}$ can be extracted, along with many interfering frequencies. It is hard to identify among all interfering frequencies. From Figure 17(a), it can be seen after LP denoising that the interfering frequencies can be reduced or eliminated to a degree, but fault frequency $f_{i}$ and rotational frequency $f_{r}$ still cannot be extracted. While the effectiveness of LP denoising method is better than WPD from the comparative analysis. It can be seen from Figure 17(b) that after AHLP denoising method, the characteristic frequencies are obvious, and the background noise is greatly reduced and eliminated. There are not many interfering frequencies in the frequency domain plot except the characteristic frequencies containing the rotational frequency $f_{r}$ and fault characteristic frequencies $f_{i}, 2$ $f_{i}$. The denoising effect of AHLP is better than LP and WPD denoising methods, which indicates the extension research of LP to AHLP is a successful exploration. Based on the displayed results and comparative analysis, it draws 


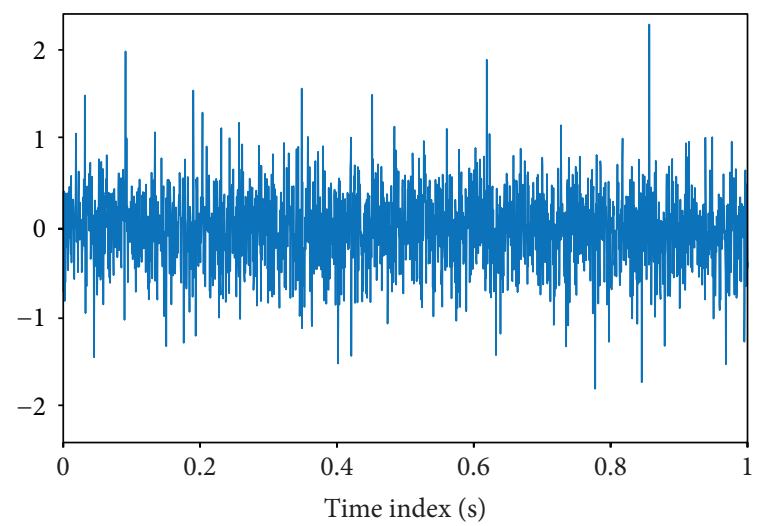

(a)

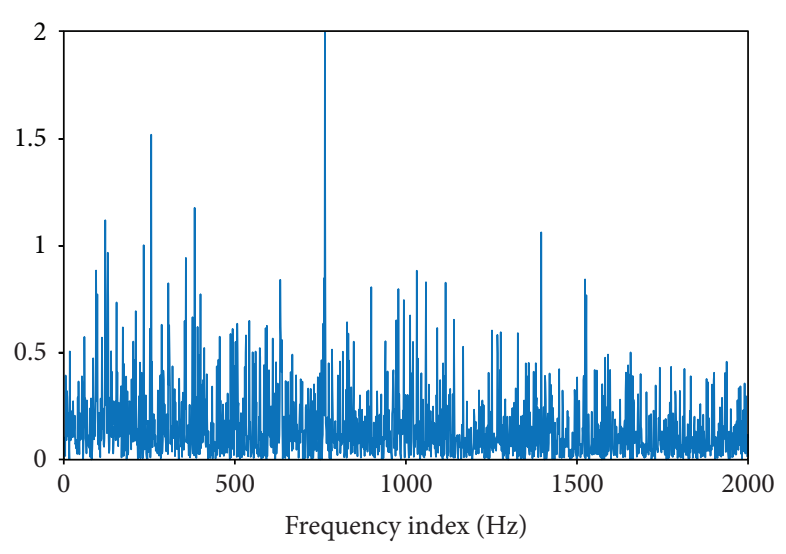

(b)

FIGURE 15: Time and frequency domain plots of collected vibration signal of IRF rolling bearing.

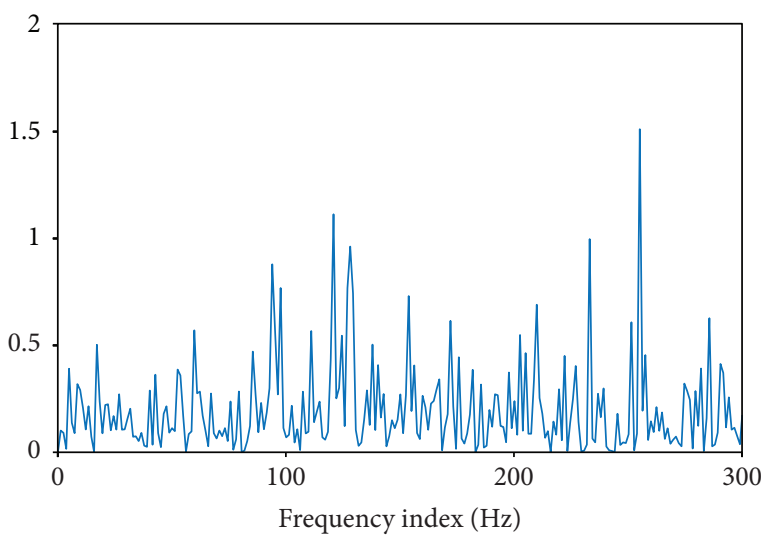

(a)

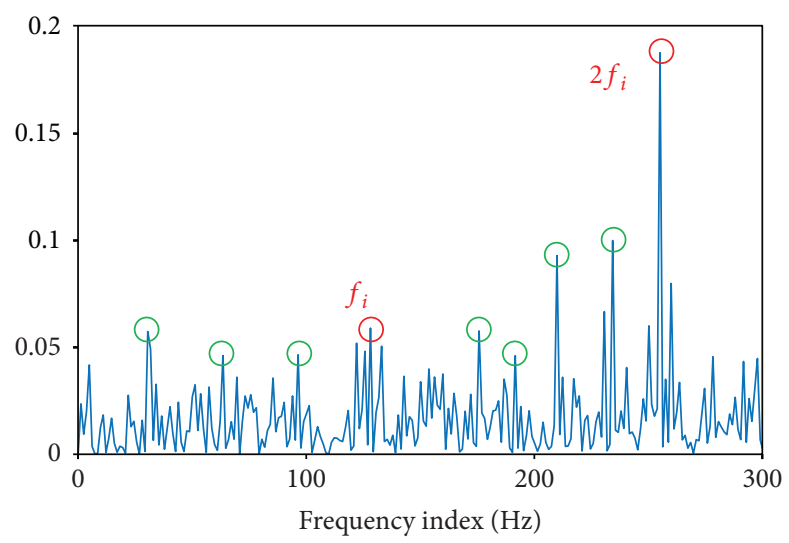

(b)

FIGURE 16: (a) Frequency domain plot of collected signal. (b) Frequency domain plot of denoised signal by WPD.

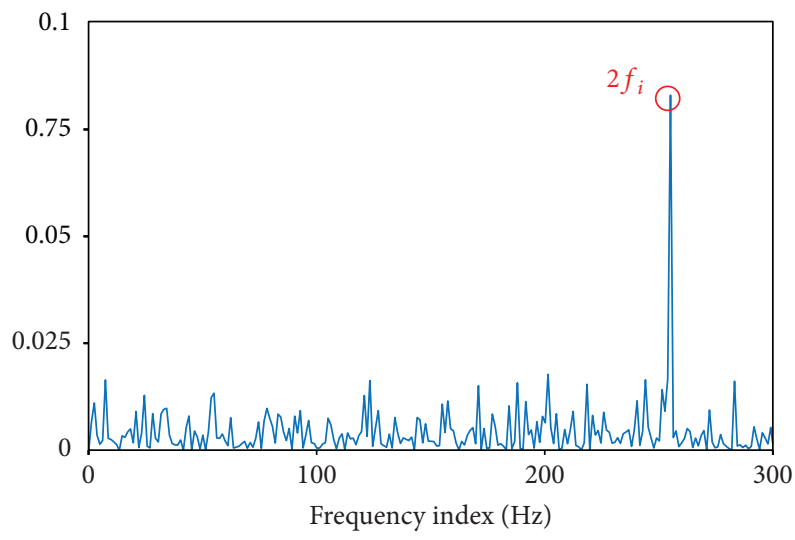

(a)

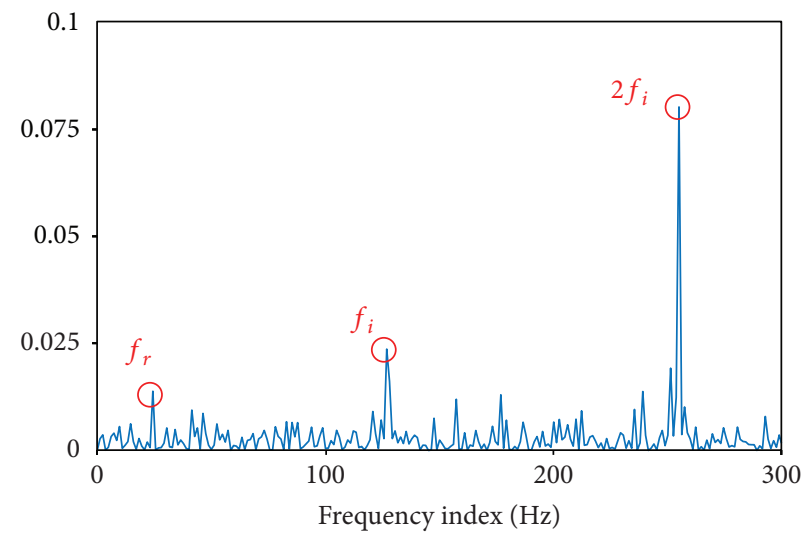

(b)

FIGURE 17: (a) Frequency domain plot of denoised signal by standard LP denoising method. (b) Frequency domain plot of denoised signal by AHLP denoising method.

a conclusion that the vibration signal of IRF rolling bearing with incipient fault can be well denoised by the proposed AHLP denoising method in practical application. It can further demonstrate the effectiveness of the proposed approach in this paper.

\section{Conclusions}

In this paper, the research work elaborates the validity and effectiveness of the proposed novel AHLP denoising approach in fault diagnosis of rolling bearing, through 
theoretical deviation, numerical simulations, and practical applications. In the field of fault detection and isolation, it is of great significance to reduce noise in vibration signal of faulty rolling bearing, to extract fault characteristic frequencies correctly and effectively. By adopting high-order polynomials to the estimate centroid of neighborhood among LP denoising method, the proposed HLP method can achieve better noise reduction effect. By choosing the optimal order among HLP denoising method aiming at vibration signals of ORF and IRF rolling bearings, the optimal denoising effect can be obtained in the proposed AHLP denoising method. The proposed method has exhibited good performance during the numerical simulations and application researches, containing simulated and practical vibration signal processing of ORF and IRF rolling bearings. In application researches, the fault characteristic frequencies can be both well extracted for the rolling bearing with severe fault corresponding to IMS bearing data processing and the rolling bearing with incipient fault corresponding to our own conducted experiment on Drivetrain Diagnostics Simulator. The displayed results and comparative analysis indicate that the proposed method can achieve good denoising results towards different degrees of faults. To sum up, the research work in this paper can demonstrate the significance and superiority of the proposed novel approach in fault diagnosis of rolling bearing.

Furthermore, this paper mainly deals with vibration signal of ORF and IRF rolling bearing, while in the field of rotary machinery, most of the denoising methods cater to various fault detections, such as gear faults and rotor faults. In our researches, gear faults have been dealt with and proper denoising effect can be achieved, so the proposed novel approach holds potential for wide employment in practical engineering applications. For the practical application in real industrial situation, the additional overhead costs concerning hardware, software, installation, and other factors should be also considered. This aspect of the proposed approach would be researched in our future work to explore its suitable application fields.

\section{Data Availability}

The authors also appreciate the free download of the original bearing failure data and one photo picture provided by Intelligent Maintenance Systems (IMS) Center of University of Cincinnati.

\section{Conflicts of Interest}

The authors declare that there are no conflicts of interest regarding the publication of this article.

\section{Acknowledgments}

This research project is supported by the National Natural Science Foundation of China under Grant no. 51475339 and no. 51105284, the Natural Science Foundation of Hubei Province under Grant no. 2016CFA042, and the
Postgraduate Overseas Visiting Scholar Fund of Wuhan University of Science and Technology.

\section{References}

[1] Y. Li, M. Xu, Y. Wei, and W. Huang, "Rotating machine fault diagnosis based on intrinsic characteristic-scale decomposition," Mechanism and Machine Theory, vol. 94, pp. 9-27, 2015.

[2] Y. Lv, R. Yuan, T. Wang, H. Li, and G. Song, "Health degradation monitoring and early fault diagnosis of a rolling bearing based on CEEMDAN and improved MMSE," Materials, vol. 11, no. 6, p. 1009, 2018.

[3] H. Liu, J. Zhang, Y. Cheng, and C. Lu, "Fault diagnosis of gearbox using empirical mode decomposition and multi-fractal detrended cross-correlation analysis," Journal of Sound and Vibration, vol. 385, pp. 350-371, 2016.

[4] R. Burdzik, L. Konieczny, and P. Folęga, "Structural health monitoring of rotating machines in manufacturing processes by vibration methods," Advanced Materials Research, vol. 1036, pp. 642-647, 2014.

[5] Y. Tian, J. Ma, C. Lu, and Z. Wang, "Rolling bearing fault diagnosis under variable conditions using LMD-SVD and extreme learning machine," Mechanism and Machine Theory, vol. 90, pp. 175-186, 2015.

[6] Y. Tian, Z. Wang, and C. Lu, "Self-adaptive bearing fault diagnosis based on permutation entropy and manifold-based dynamic time warping," Mechanical Systems and Signal Processing, vol. 114, pp. 658-673, 2019.

[7] X. Wang, Y. Zi, and Z. He, "Multiwavelet denoising with improved neighboring coefficients for application on rolling bearing fault diagnosis," Mechanical Systems and Signal Processing, vol. 25, no. 1, pp. 285-304, 2011.

[8] H. Liu and M. Han, "A fault diagnosis method based on local mean decomposition and multi-scale entropy for roller bearings," Mechanism and Machine Theory, vol. 75, pp. 67-78, 2014.

[9] Y. Lv, R. Yuan, and G. Song, "Multivariate empirical mode decomposition and its application to fault diagnosis of rolling bearing," Mechanical Systems and Signal Processing, vol. 81, pp. 219-234, 2016.

[10] Y. Li, M. Xu, H. Zhao, and W. Huang, "Hierarchical fuzzy entropy and improved support vector machine based binary tree approach for rolling bearing fault diagnosis," Mechanism and Machine Theory, vol. 98, pp. 114-132, 2016.

[11] M. Cao and P. Qiao, "On the wavelet-fractal nonlinear damage diagnosis of mechanical systems," Smart Materials and Structures, vol. 18, no. 8, article 085022, 2009.

[12] A. Tabrizi, L. Garibaldi, A. Fasana, and S. Marchesiello, "Early damage detection of roller bearings using wavelet packet decomposition, ensemble empirical mode decomposition and support vector machine," Meccanica, vol. 50, no. 3, pp. $865-$ 874, 2015.

[13] H. Ocak, K. A. Loparo, and F. M. Discenzo, "Online tracking of bearing wear using wavelet packet decomposition and probabilistic modeling: a method for bearing prognostics," Journal of Sound and Vibration, vol. 302, no. 4-5, pp. 951-961, 2007.

[14] Y. Pan, J. Chen, and X. Li, "Bearing performance degradation assessment based on lifting wavelet packet decomposition and fuzzy c-means," Mechanical Systems and Signal Processing, vol. 24, no. 2, pp. 559-566, 2010. 
[15] Z. Qiao and Z. Pan, "SVD principle analysis and fault diagnosis for bearings based on the correlation coefficient," Measurement Science and Technology, vol. 26, no. 8, article 085014, 2015.

[16] H. Jiang, J. Chen, G. Dong, T. Liu, and G. Chen, "Study on Hankel matrix-based SVD and its application in rolling element bearing fault diagnosis," Mechanical Systems and Signal Processing, vol. 52-53, pp. 338-359, 2015.

[17] W. Guo and P. W. Tse, "A novel signal compression method based on optimal ensemble empirical mode decomposition for bearing vibration signals," Journal of Sound and Vibration, vol. 332, no. 2, pp. 423-441, 2013.

[18] F. Takens, Detecting Strange Attractors in Turbulence/l Dynamical Systems and Turbulence, Warwick 1980, Springer, Berlin, Heidelberg, 1981.

[19] M. B. Kennel, R. Brown, and H. D. I. Abarbanel, "Determining embedding dimension for phase-space reconstruction using a geometrical construction," Physical Review A, vol. 45, no. 6, pp. 3403-3411, 1992.

[20] D. M. Walker and N. B. Tufillaro, "Phase space reconstruction using input-output time series data," Physical Review E, vol. 60, no. 4, pp. 4008-4013, 1999.

[21] C. A. L. Pires and A. Hannachi, "Independent subspace analysis of the sea surface temperature variability: non-Gaussian sources and sensitivity to sampling and dimensionality," Complexity, vol. 2017, Article ID 3076810, 23 pages, 2017.

[22] I. Stojkovic and Z. Obradovic, "Sparse learning of the disease severity score for high-dimensional data," Complexity, vol. 2017, Article ID 7120691, 11 pages, 2017.

[23] M. P. Mareca and B. Bordel, "Improving the complexity of the Lorenz dynamics," Complexity, vol. 2017, Article ID 3204073, 16 pages, 2017.

[24] D. Chelidze and W. Zhou, "Smooth orthogonal decomposition-based vibration mode identification," Journal of Sound and Vibration, vol. 292, no. 3-5, pp. 461-473, 2006.

[25] D. Chelidze and G. Chelidze, "Nonlinear model reduction based on smooth orthogonal decomposition," in Proceedings of the Ninth IASTED International Conference on Control and Applications, pp. 325-330, ACTA Press, Montreal, Quebec, 2007.

[26] S. Ilbeigi and D. Chelidze, "Persistent model order reduction for complex dynamical systems using smooth orthogonal decomposition," Mechanical Systems and Signal Processing, vol. 96, pp. 125-138, 2017.

[27] P. Grassberger, R. Hegger, H. Kantz, C. Schaffrath, and T. Schreiber, "On noise reduction methods for chaotic data," Chaos: An Interdisciplinary Journal of Nonlinear Science, vol. 3, no. 2, pp. 127-141, 1993.

[28] W. F. He, L. Cheng, X. G. Li, and J. Luo, "Nonlinear noise reduction and its application to the vibration signal analysis," Journal of Vibration Engineering, vol. 17, pp. 408-411, 2004.

[29] T. Sauer, "A noise reduction method for signals from nonlinear systems," Physica D: Nonlinear Phenomena, vol. 58, no. 1-4, pp. 193-201, 1992.

[30] X. Wan, K. Iwata, J. Riera, T. Ozaki, M. Kitamura, and R. Kawashima, "Artifact reduction for EEG/fMRI recording: nonlinear reduction of ballistocardiogram artifacts," Clinical Neurophysiology, vol. 117, no. 3, pp. 668-680, 2006.

[31] N. Jevtić, P. Stine, and J. S. Schweitzer, "Nonlinear time series analysis of Kepler space telescope data: mutually beneficial progress," Astronomische Nachrichten, vol. 333, no. 10, pp. 983-986, 2012.

[32] L. Qian, J. Chen, and X. Feng, "Local projection stabilized and characteristic decoupled scheme for the fluid-fluid interaction problems," Numerical Methods for Partial Differential Equations, vol. 33, no. 3, pp. 704-723, 2017.

[33] N. Jevtic, J. S. Schweitzer, and P. Stine, “Optimizing nonlinear projective noise reduction for the detection of planets in mean-motion resonances in transit light curves," Chaos Theory, pp. 191-198, 2011.

[34] R. Cawley and G. H. Hsu, "Local-geometric-projection method for noise reduction in chaotic maps and flows," Physical Review A, vol. 46, no. 6, pp. 3057-3082, 1992.

[35] R. Hegger, H. Kantz, and T. Schreiber, "Practical implementation of nonlinear time series methods: the TISEAN package," Chaos: An Interdisciplinary Journal of Nonlinear Science, vol. 9, no. 2, pp. 413-435, 1999.

[36] J. M. Moore, M. Small, and A. Karrech, "Improvements to local projective noise reduction through higher order and multiscale refinements," Chaos: An Interdisciplinary Journal of Nonlinear Science, vol. 25, no. 6, article 063114, 2015.

[37] A. Cordero, E. Gómez, and J. R. Torregrosa, "Efficient highorder iterative methods for solving nonlinear systems and their application on heat conduction problems," Complexity, vol. 2017, Article ID 6457532, 11 pages, 2017.

[38] H. Kantz and T. Schreiber, Nonlinear Time Series Analysis, Cambridge University Press, 2004.

[39] L. Cao, "Practical method for determining the minimum embedding dimension of a scalar time series," Physica D: Nonlinear Phenomena, vol. 110, no. 1-2, pp. 43-50, 1997.

[40] J. Antoni and R. B. Randall, "A stochastic model for simulation and diagnostics of rolling element bearings with localized faults," Journal of Vibration and Acoustics, vol. 125, no. 3, pp. 282-289, 2003.

[41] R. B. Randall and J. Antoni, "Rolling element bearing diagnostics-a tutorial," Mechanical Systems and Signal Processing, vol. 25, no. 2, pp. 485-520, 2011.

[42] R. B. Randall, J. Antoni, and S. Chobsaard, "The relationship between spectral correlation and envelope analysis in the diagnostics of bearing faults and other cyclostationary machine signals," Mechanical Systems and Signal Processing, vol. 15, no. 5, pp. 945-962, 2001.

[43] J. Antoni and R. B. Randall, "Differential diagnosis of gear and bearing faults," Journal of Vibration and Acoustics, vol. 124, no. 2, pp. 165-171, 2002.

[44] Y. N. Pan, J. Chen, and X. L. Li, "Spectral entropy: a complementary index for rolling element bearing performance degradation assessment," Proceedings of the Institution of Mechanical Engineers, Part C: Journal of Mechanical Engineering Science, vol. 223, no. 5, pp. 1223-1231, 2009.

[45] H. Qiu, J. Lee, J. Lin, and G. Yu, "Wavelet filter-based weak signature detection method and its application on rolling element bearing prognostics," Journal of Sound and Vibration, vol. 289, no. 4-5, pp. 1066-1090, 2006. 


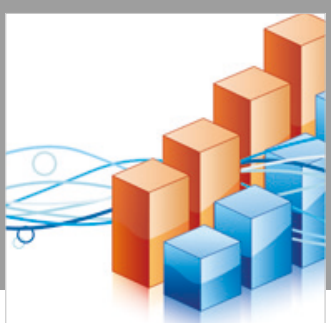

Advances in

Operations Research

\section{-n-m}
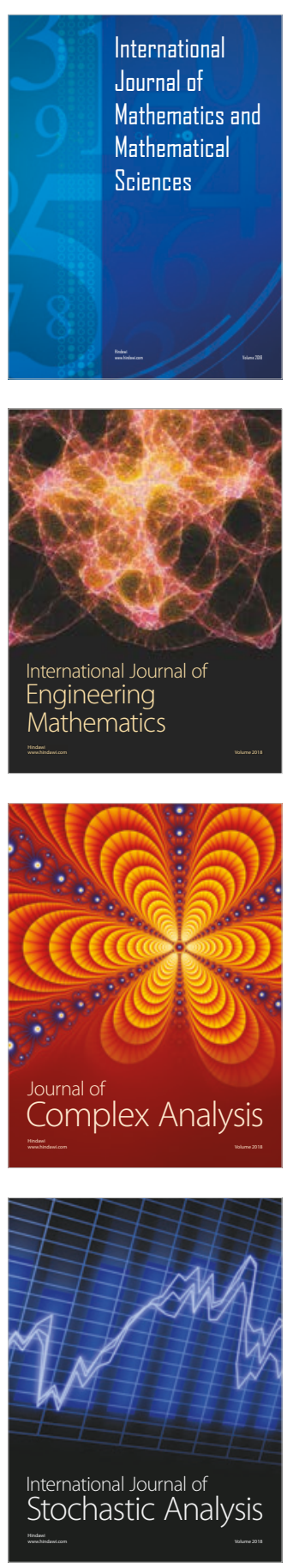
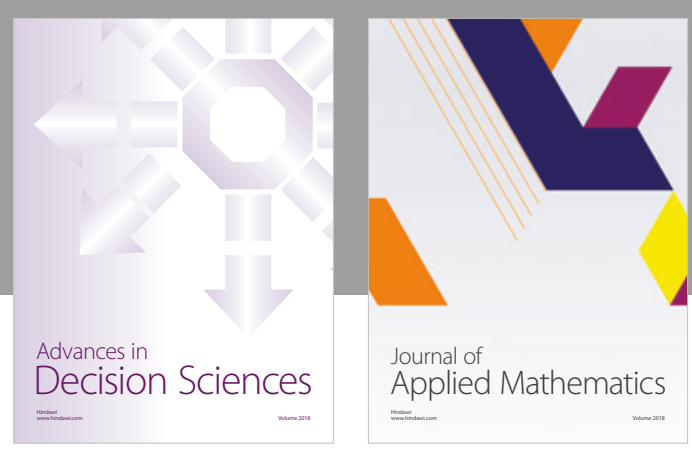

Journal of

Applied Mathematics
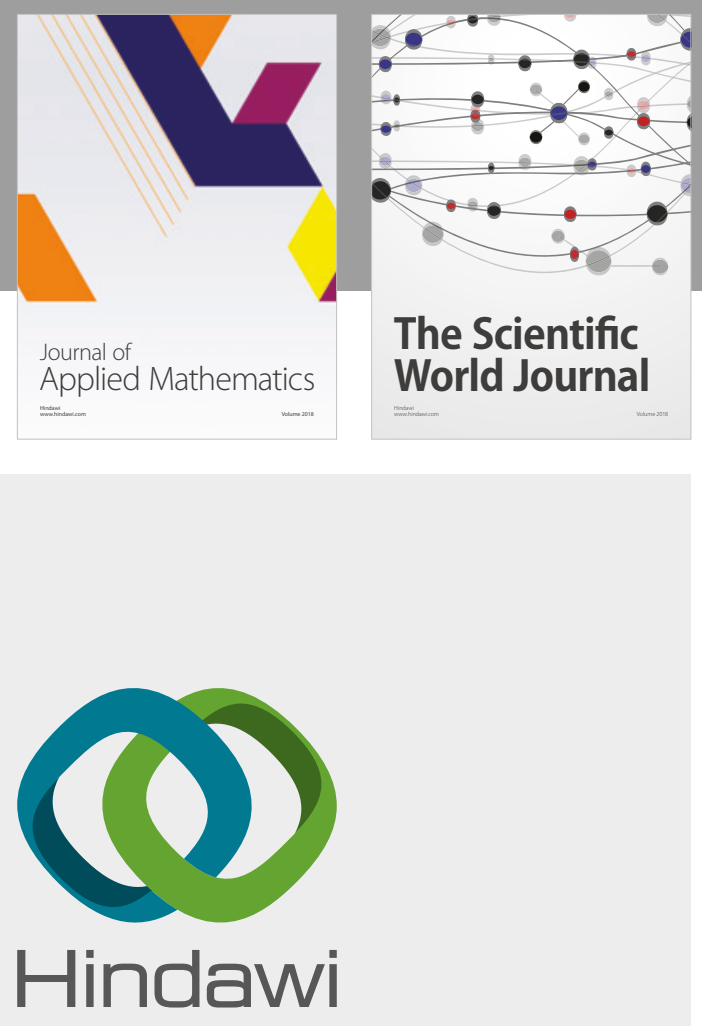

Submit your manuscripts at

www.hindawi.com

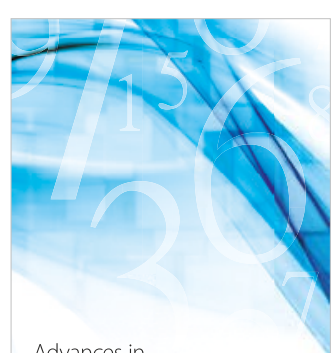

Advances in
Numerical Analysis
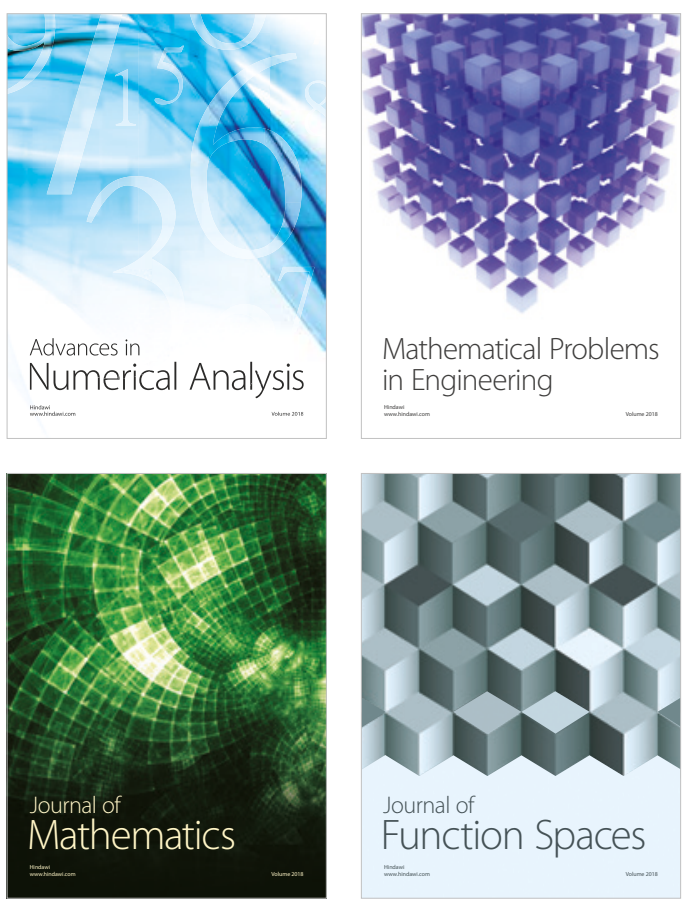

Mathematical Problems in Engineering

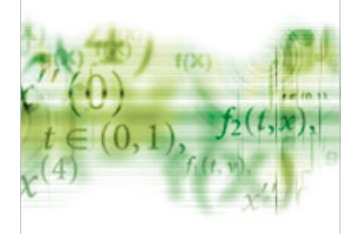

International Journal of

Differential Equations

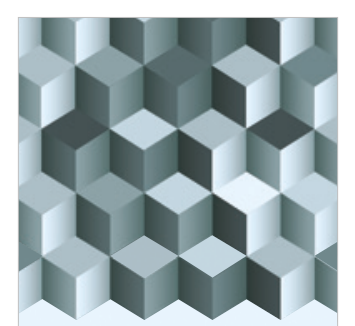

Journal of

Function Spaces
The Scientific

World Journal

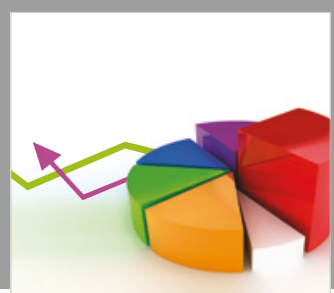

Journal of

Probability and Statistics
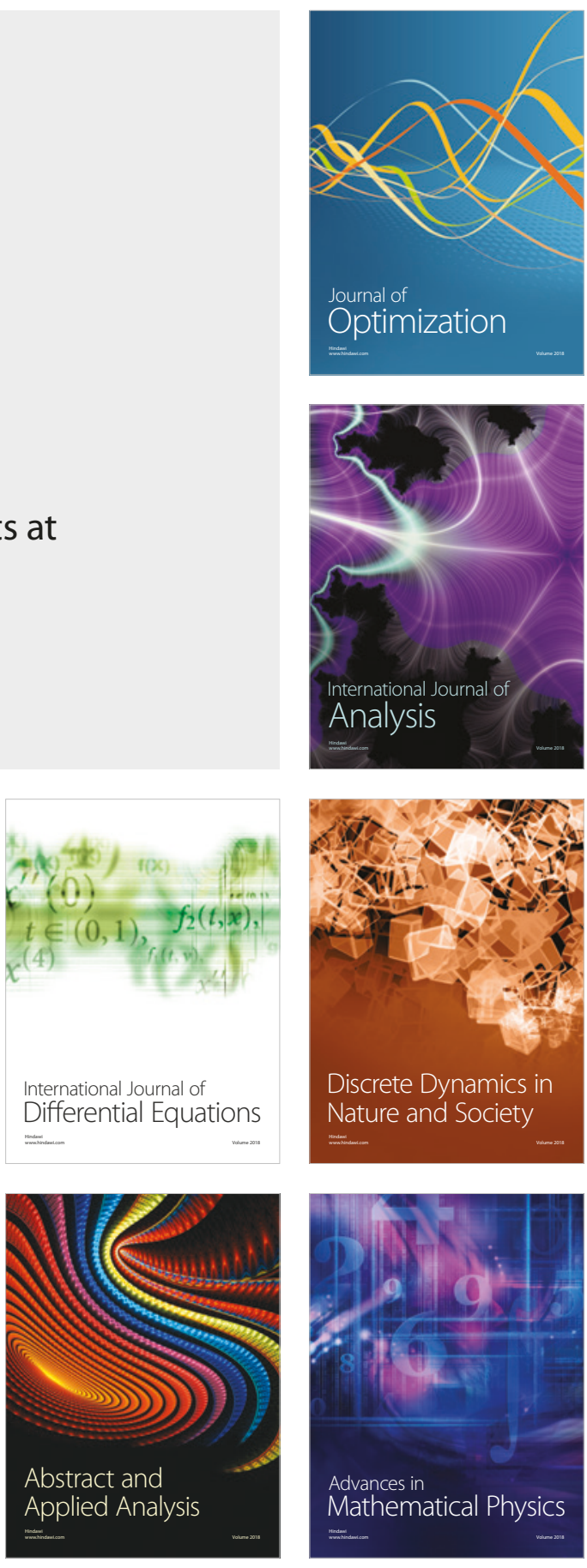